\title{
Supporting Information for Modeling the Mechanical Response of Microtubule Lattices to Pressure
}

Lukasz Szatkowski ${ }^{\dagger, \neq}$, Rohith Anand Varikoti ${ }^{\dagger}$, Ruxandra I Dima ${ }^{*}+$

${ }^{\dagger}$ Department of Chemistry, University of Cincinnati, Cincinnati, OH 45221

${ }^{\ddagger}$ Division of Science, Mathematics, and Engineering, University of South Carolina Sumter, SC 29150

Table of Contents:

I. Data Analysis 


\section{Data Analysis}

\section{$\underline{\text { I.1 Additional analysis for the Full Atomistic MD simulations }}$}

\section{Energy Analysis}

For each production run corresponding to an MT fragment we used a NAMD $[1,2]$ based script to extract the Coulombic, van der Waals, and total energy of the 4 central monomers: the central dimer (red circle in Figure S2) and its adjacent tubulin monomers (blue circles in Figure S2. For the models with PEG(50) and PEG(50)R41, the energy of the PEG molecules was not included in the calculations. Only energies between the same atoms in all systems (with and without PEGs) were evaluated.

We plotted the average energy data from the last $10 \mathrm{~ns}$ (as moving average with \pm 5 points) of each trajectory (see Figure S7) and we calculated the average values of the Coulombic, van der Waals, and total energy from the last 2 ns of the production runs for each system (see Table S3). Finally, we compared the average energies from the last 2 ns between the system without and with PEGs by calculating the relative change of the energy of each system with PEG molecules vs. the reference system without PEG (e.g. energy with PEG(50) - without PEG)) as a percent of the energy of the reference system. Positive values of the change in energy mean that the interactions are favorable (more negative), while negative values mean an increase in the energy of the system in the presence of PEG molecules.

\section{Water molecules at the lateral interface}

Water molecules were counted in VMD [3] as all waters found within $3.5 \AA$ from the central dimer and, at the same time, within the same distance from the adjacent tubulin monomers (see Figure S10). We counted the number of water molecules for each frame of the production runs for each MT system and then we extracted the average number of water molecules for the last 2 ns of the production run (Table S4).

\section{Contacts Analysis}

We analyzed the contacts between any two adjacent tubulin monomers (from the 2 protofilament fragments) for each frame in the last 2 ns of a production run, using VMD [3]. A contact between two residues was counted if at least one pair of heavy atoms from their side-chains ( $\mathrm{C}, \mathrm{N}, \mathrm{O}$ and $\mathrm{S}$ atoms) was within $5.2 \AA$. We calculated the averages and standard deviations of the number of contacts (for the last 2 ns of the production runs) for each system (see Table S4). 
The average structure for each system (without water and ions) was created in VMD [3] for the last $2 \mathrm{~ns}$ of each production run. The change in the position of each heavy atom of PEG(50) (see Table S5) was calculated in VMD [3] as the difference between its $X Y Z$ coordinates in the average structure and in the initial structure resulting from docking. For both systems (AWAY+PEG(50) and SEAM+PEG(50)) a positive change in the displacement on the $X$-axis indicates the shift of the position of the PEG(50) atom away from the dimer (toward adjacent tubulin monomers: $a 46$ and $\beta 46$ for AWAY+PEG(50), and $\beta 52$ and $a 65$ for SEAM+PEG(50); see Figure S2). A positive value for the $Y$-axis displacement indicates a shift away from the MT surface, and a positive displacement on the $Z$-axis indicates a shift toward the + end of the MT (see Figure 5 in the main text and Figure S13). The total displacement for each heavy atom was calculated as the amplitude of the vector difference between the initial position of the atom and its position in the average structure.

\section{$\underline{\text { I.2 Relative native contacts (RQn) and first breaking force }}$}

For each trajectory from our MT compression studies, we collected the force vs. time and vs. depth (see Figure S14). We used this data, together with the analysis of the relative native contacts, to find the frame at which the first lateral break between two protofilaments happens. The force corresponding to this frame is the first breaking force (FBF).

To find out whether the interface is broken or not we evaluated the number of native contacts between two neighboring protofilaments (lateral break) and between dimers within each protofilament (longitudinal break) within a cut-off distance of $13 \AA$ during the trajectory, following our previous studies. [3] Beyond the $13 \AA$ distance, the Lennard-Jones attractive potential term in Eqn. (1) from the main text becomes negligible. The contacts were divided into four groups: contacts between dimers along the same protofilament, north ( $N$ ) toward + end and south (S) toward - end for the longitudinal contacts, and contacts between two protofilaments east (E) the initial one and the one on the protofilament with index $n+1$, and west (W) the initial one and the one on the protofilament with index $\mathrm{n}-1$ for the lateral contacts (see Figure S1 for further explanations). For every frame of a trajectory, we evaluate if the initial native contacts are still within the cutoff distance and then we count the number of these contacts for each dimer in each direction. Then we summarized the contact numbers for all dimers in a single protofilament and divided by the total number of the interfaces to get the average number of contacts for each protofilament at each frame. For example, for 8 dimers long MT along each protofilament there are 7 longitudinal interfaces (in both $\mathrm{N}$ and $S$ directions), 8 full interfaces (E/W) for any protofilament to protofilament interaction which is not the SEAM and 6.5 interfaces for the SEAM. Finally, we divided the average number by the initial average number of contacts at frame 0 to get the RQn 
value (for lateral: 61 per interface, for longitudinal: 69 per interface, and for the SEAM: 42 per interface) and plotted it vs. frame number, as shown in Figure S21. We focused on any sharp changes in the RQn value to find the exact frame at which a break occurs: if the number of contacts for a dimer drops to zero it signals a break (Figure S21). Then, from the force vs. frame plot, we extracted the corresponding FBF value. 


\section{Tables}

Table S1 List of MD simulations for MT fragments with and without PEG 2000 molecules. A_REF - AWAY reference system; A_P50 - AWAY with PEG(50) molecule; A_R41 - AWAY with PEG(50)R41; S_REF - SEAM reference system; S_P50) - SEAM with PEG(50) molecule; S_R41 - SEAM with PEG(50)R41; Traj\# - number of production trajectories.

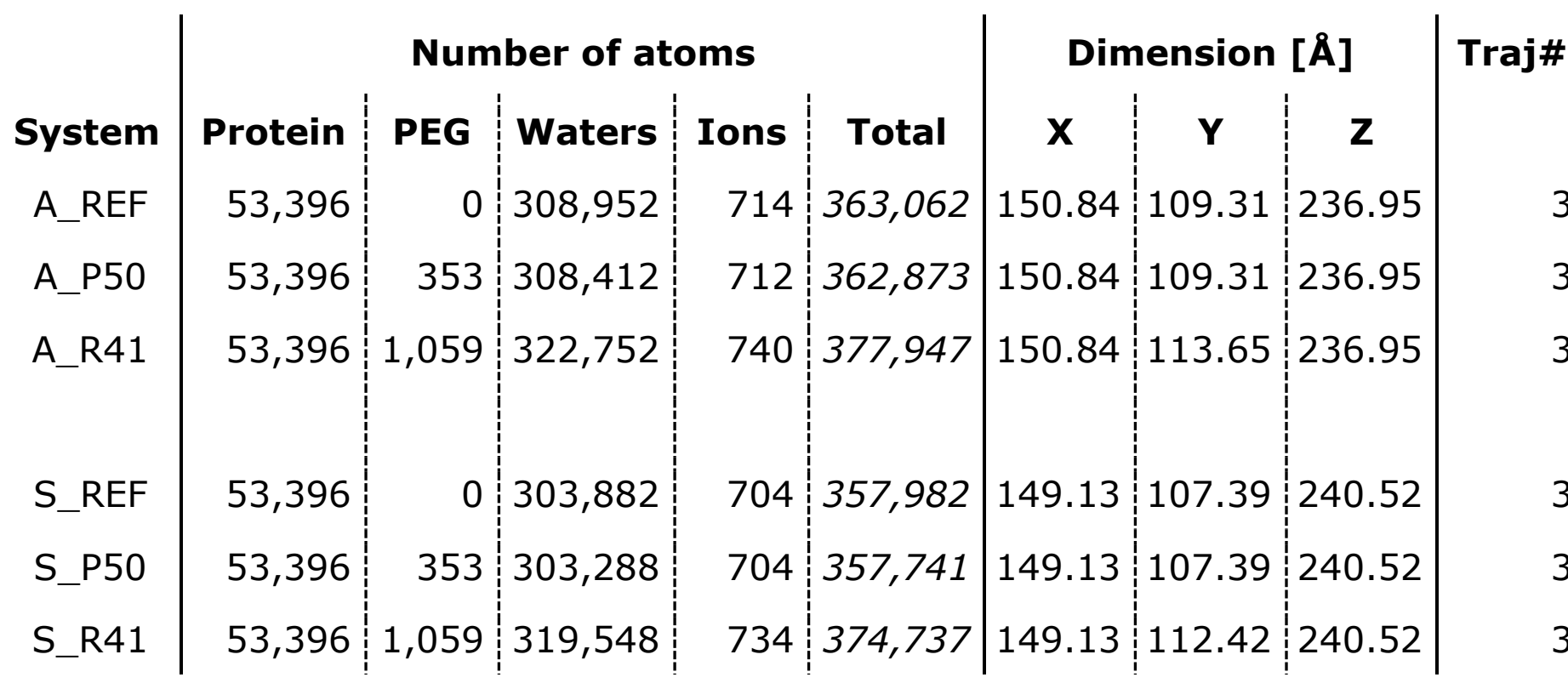

Table S2 Number of trajectories for the compression studies of each MT system. 13MTX\# length of the MT model, ST - small tip (10nm); LT - large tip (75 nm); BT - big tip (100nm); P\# - protofilament number

\begin{tabular}{lcccccccc} 
Model & Tip size & Position & \multicolumn{7}{c}{ Loading rate $[\mu \mathrm{m} / \mathrm{s}]$} \\
& & & 2.000 & 1.000 & 0.500 & 0.250 & 0.200 & 0.125 \\
13 MTx8 & BT & P1/P13 & 6 & 3 & 3 & 3 & 3 & 3 \\
13 MTx8 & BT & P5/P6 & 3 & 3 & 3 & 3 & 3 & 3 \\
13 MTx8 & BT & P6/P7 & 6 & 3 & 3 & 3 & 3 & 3 \\
13 MTx8 & ST & P5/P6 & 3 & 3 & 3 & 3 & 3 & 3 \\
13 MTx8 & ST & P6/P7 & 3 & 3 & 3 & 3 & 3 & 3 \\
13 MTx6 & LT & P5/P6 & 1 & 1 & 1 & 1 & - & 1 \\
13 MTx12 & BT & P1/P13 & 3 & 2 & 2 & 2 & - & 3 \\
13 MTx12 & BT & P5/P6 & 1 & 1 & 1 & 1 & - & 1 \\
$13 M T x 12$ & BT & P6/P7 & 3 & 2 & 2 & 2 & - & 3
\end{tabular}


Table $\mathbf{5 3}$ The average values (with standard deviation) of the Coulomb and van der Waals energy for a full dimer and adjacent tubulin monomers on the AWAY and SEAM interfaces for systems without PEG, with PEG(50), and with PEG(50)R41 molecules. The values were calculated for the last $2 \mathrm{~ns}$ of the production runs and are given in $[\mathrm{kcal} / \mathrm{mol}]$. The relative change (energy with e.g. PEG(50) - without PEG)) of the energy of the dimer and adjacent tubulin monomers is given in percentages. A positive value means that the interactions are favorable (more negative), while a negative value signals an increase in the energy.

Coulomb

$\begin{array}{lccc} & & \text { AWAY } \\ \text { without PEG } & -30332.49 \pm 100.82 & -7271.53 \pm 37.17 \\ \text { with PEG(50) } & -30706.14 \pm 96.32 & -7274.79 \pm 36.27 \\ \text { change }[\%] & +1.23 \% & +0.04 \% \\ \text { with PEG(50)R41 } & -30698 \pm 117.08 & -7301.83 \pm 47.90 \\ \text { change }[\%] & +1.21 \% & +0.42 \% \\ & & \text { SEAM } & \\ \text { without PEG(50) } & -29919.63 \pm 120.27 & -7216.37 \pm 35.90 \\ \text { with PEG(50) } & -29710.27 \pm 112.22 & -7228.62 \pm 37.48 \\ \text { change }[\%] & -0.70 \% & -0.17 \% \\ \text { with PEG(50)R41 } & -30284.37 \pm 135.64 & -7218.72 \pm 36.44 \\ \text { change }[\%] & +1.22 \% & +0.03 \%\end{array}$

Table S4 The average number of contacts and average number of water molecules located between adjacent tubulin monomers from the 2 protofilaments. The average is taken over the last 2 ns of production runs. Contacts calculated for all heavy atoms ( $, N, O$ and S) from side chains within $5.2 \AA$. Water molecules are counted only if they are within $3.5 \AA$ from two adjacent tubulin monomers (see Figure S10).

\section{Contacts}

\begin{tabular}{lcll} 
AWAY: & $39.27 \pm 5.05$ & SEAM: & $37.23 \pm 6.61$ \\
AWAY + PEG(50): & $44.74 \pm 5.25$ & SEAM + PEG(50): & $37.26 \pm 3.67$ \\
AWAY + PEG(50)R41: & $48.90 \pm 3.54$ & SEAM + PEG(50)R41: & $34.98 \pm 5.77$ \\
& \multicolumn{2}{c}{ Water molecules } \\
AWAY: & $209.12 \pm 20.90$ & SEAM: & $156.16 \pm 17.42$ \\
AWAY + PEG(50): & $221.63 \pm 24.52$ & SEAM + PEG(50): & $175.83 \pm 18.09$ \\
AWAY + PEG(50)R41: & $218.19 \pm 13.71$ & SEAM + PEG(50)R41: & $145.91 \pm 20.39$
\end{tabular}


Table S5 List of the heavy atoms (C, O) from PEG(50)/PEG(50)R41 with their indexes in PEG(50) and PEG(50)R41 molecules

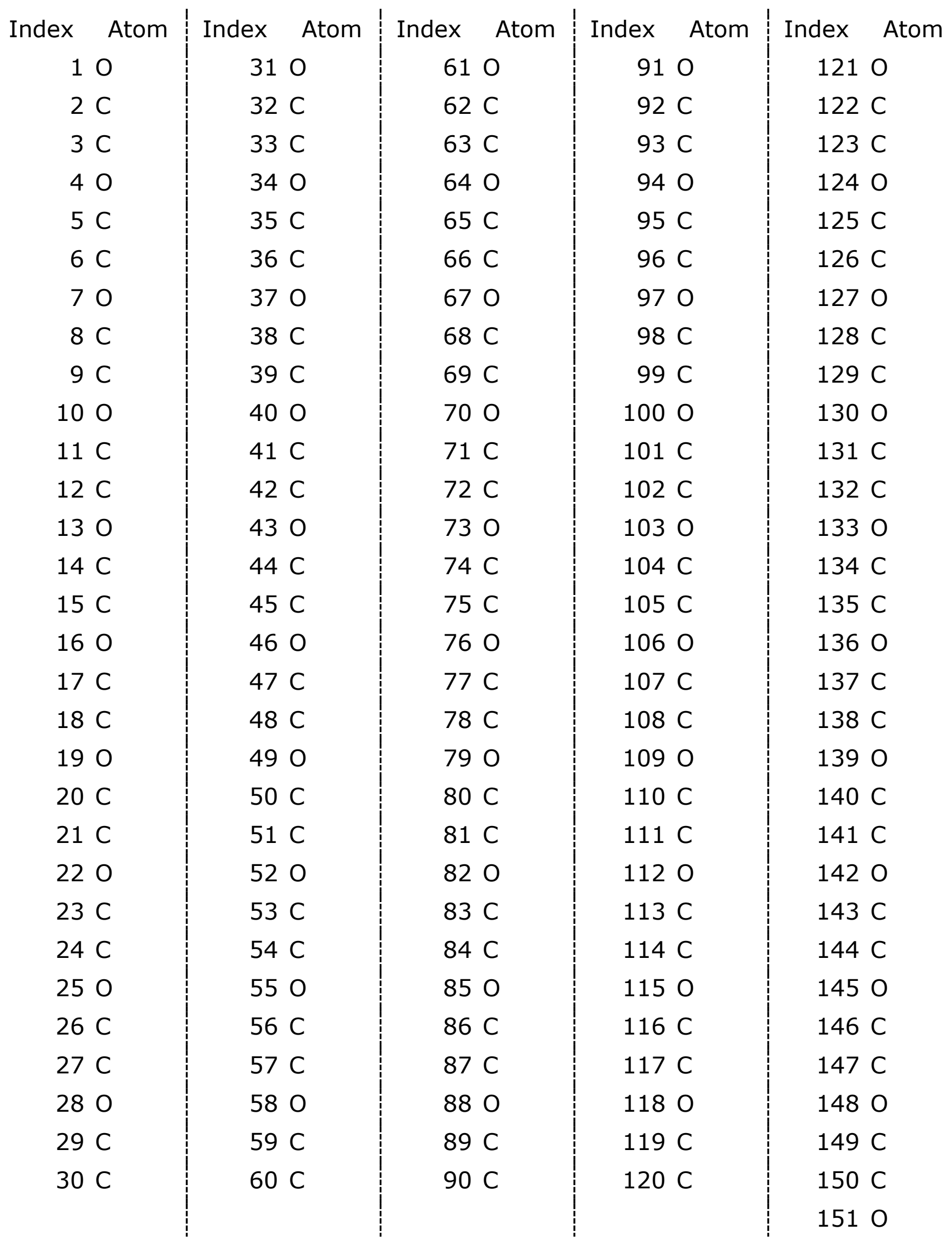




\section{Figures}
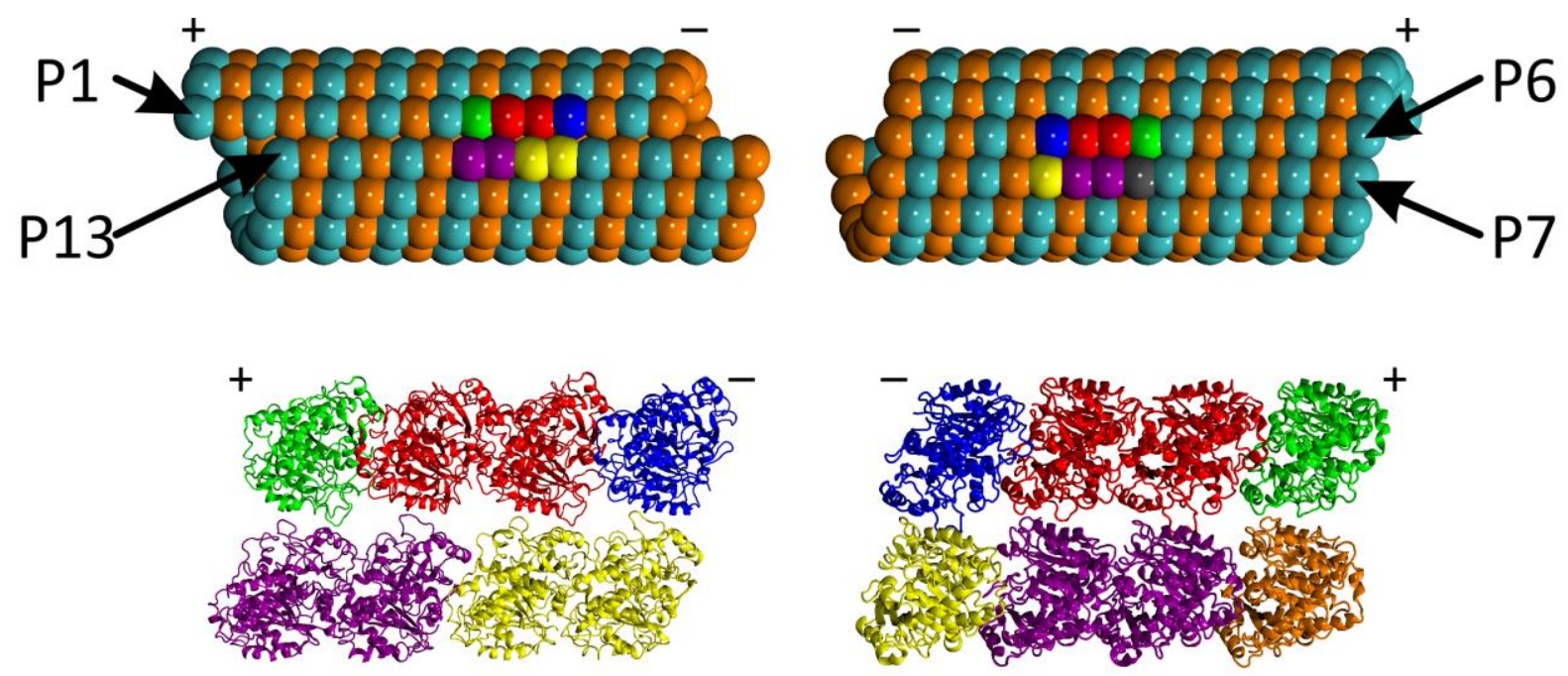

Figure S1 Top: Schematics of an MT filament with 13 protofilaments and 8 dimers in length (atubulin is orange; $\beta$-tubulin is cyan; for simplification, each monomer is represented by one sphere). The two MT fragments used for docking of PEGs are indicated: SEAM is on the left hand side, and AWAY is on the right hand side. The + and - ends of the MT are labeled. Different colors are used to indicate full dimers/monomers for each system (MT fragment) used for docking. Full dimers have the same color for the $a$ and $\beta$ tubulin monomers. Arrows show the selected protofilaments (P1 - protofilament 1; P6 - protofilament 6; P7 - protofilament 7, P13 protofilament 13). Bottom: Cartoon representation of the selected 2x4 MT matrices. Contacts: north $(\mathrm{N})$ contact between two dimers in the direction toward the + end, for example between the red and green monomers; south (S) contact between two dimers in the direction toward the - end, for example between the red and blue monomers, east (E) contact between two dimers in the same ring, for example the red and magenta for AWAY and the red and magenta/yellow for the SEAM, and west (W) contact in the opposite direction compared to the (E) contact.
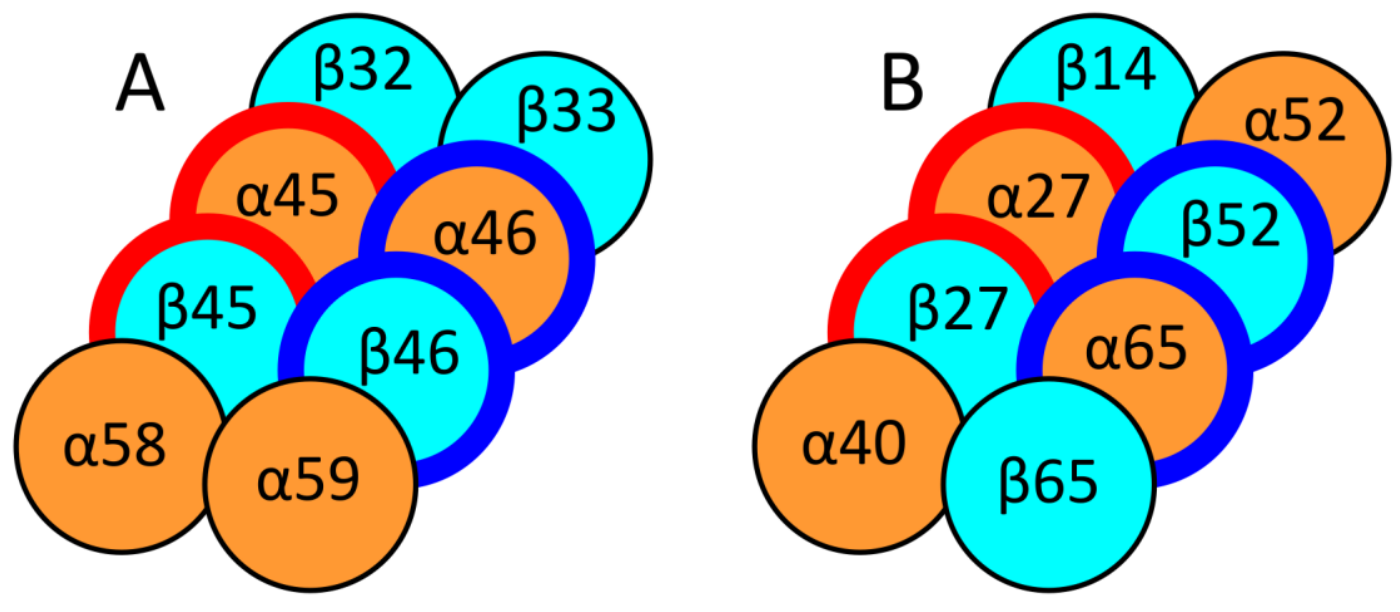

Figure $\mathbf{S 2}$ Cartoon depiction of the position of monomers for the AWAY (A) and SEAM (B) models. P6 is on the left, and P7 on the right in A. P1 is on the left, and P13 is on the right in B. Red and blue circles indicate monomer pairs or dimers used in our analysis. 

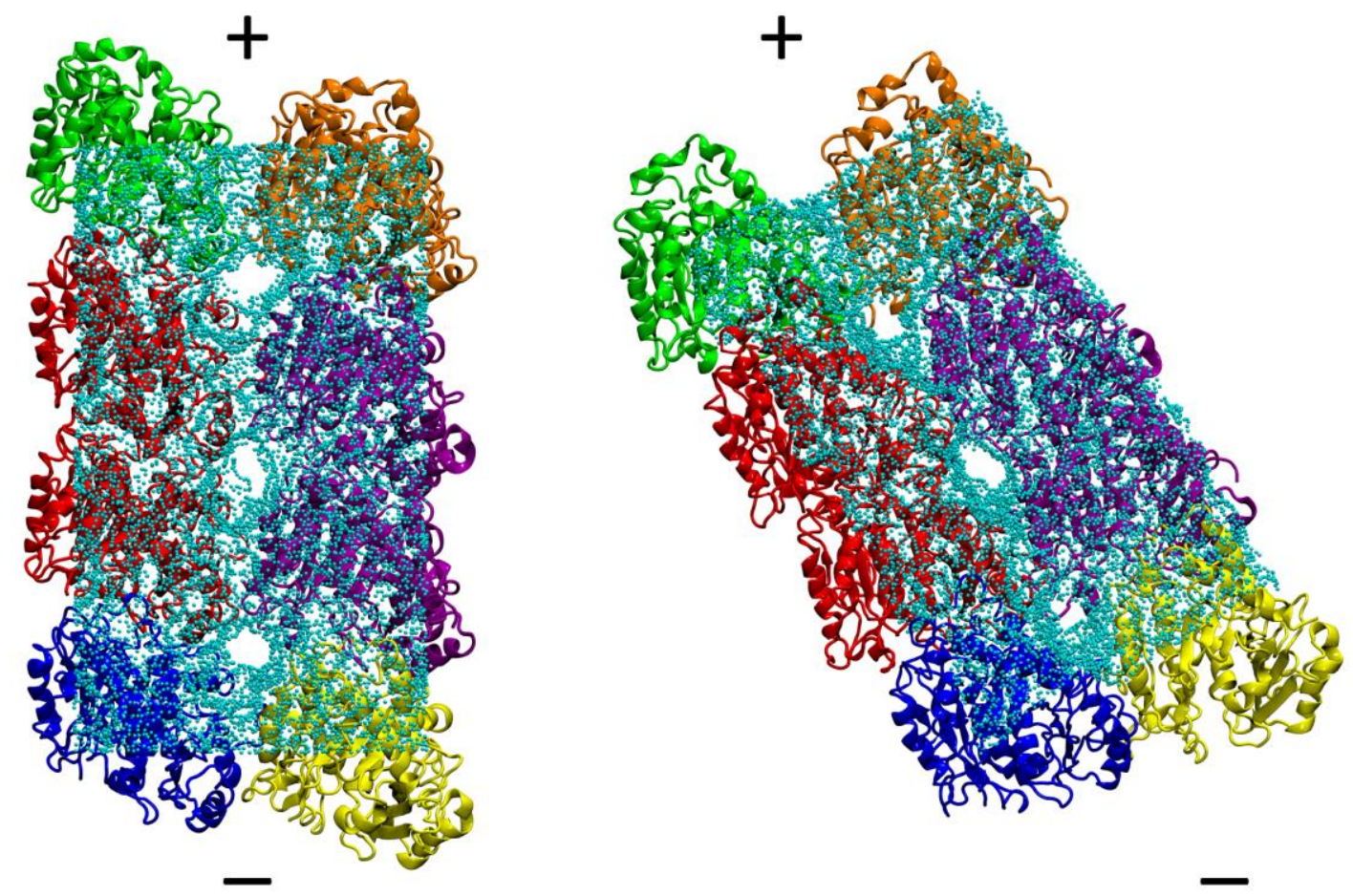

Figure S3 The AWAY system with the color coding for each dimer/monomer following Figure S1. The 9999 randomly selected docking grid points used in BUDE (three different sets with randomly selected 9999 grid points were used for docking for both systems: SEAM and AWAY) are also shown. Left hand side: view from the top of the MT. Right hand side: top-down view. P6 is on the left, P7 on the right, and + and - ends of the MT are labeled.

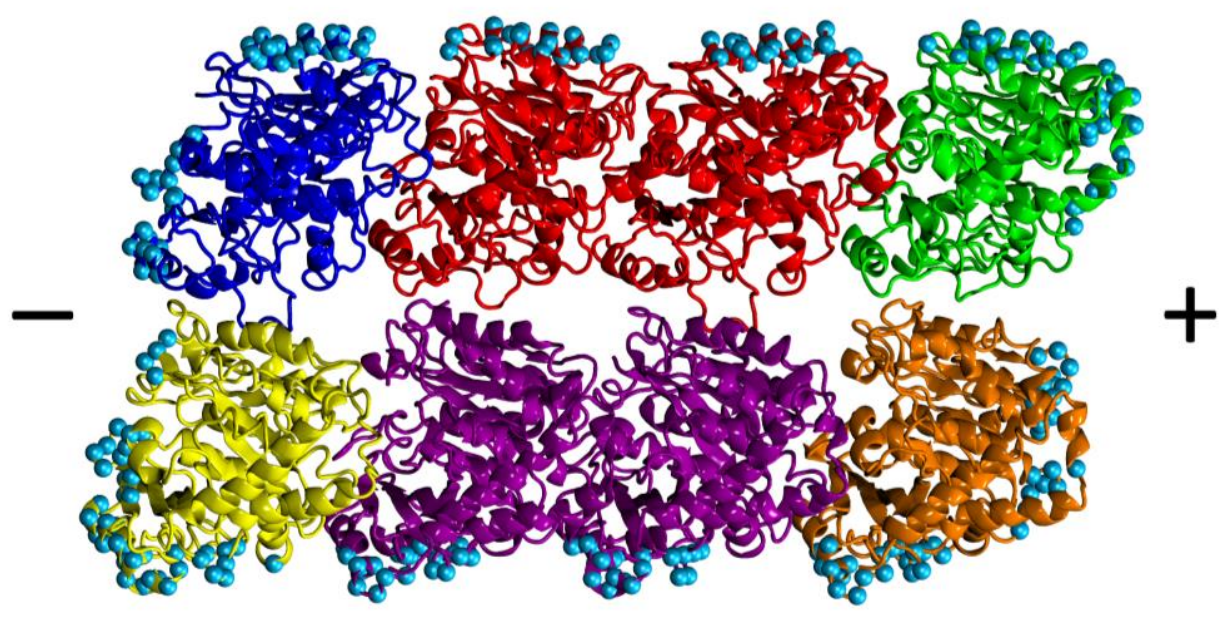

Figure S4 The AWAY reference system (without PEG(50) molecule, water and ion omitted for clarity) for the MD simulation runs. Each dimer/monomer colors follow Figure S1. The frozen Ca atoms are indicated with blue spheres. The + and - ends of the MT are labeled. A similar pattern of frozen atoms was selected for the SEAM system, and for the MT systems with PEG(50) molecules. 

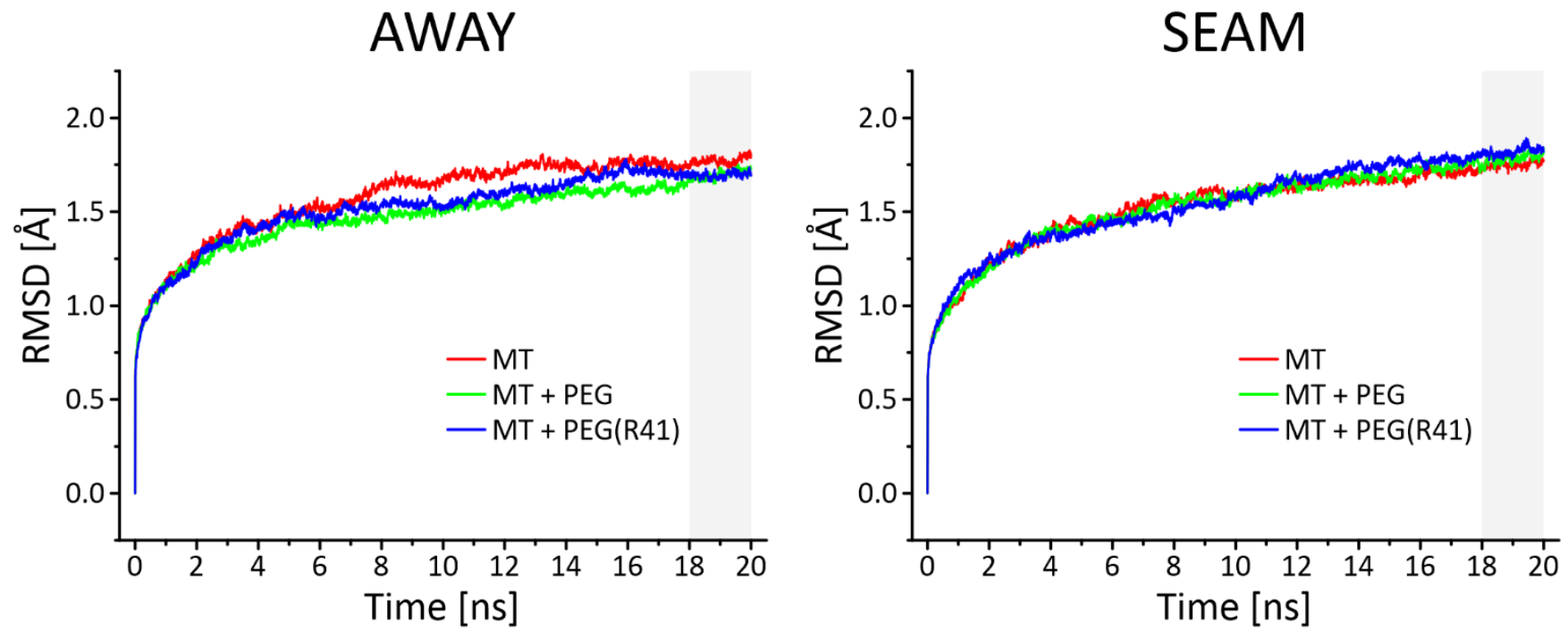

Figure S5 Average MT backbone RMSD plots from all production runs for AWAY (left) and SEAM (right) models. Gray areas indicate the last 2 ns of the trajectories.
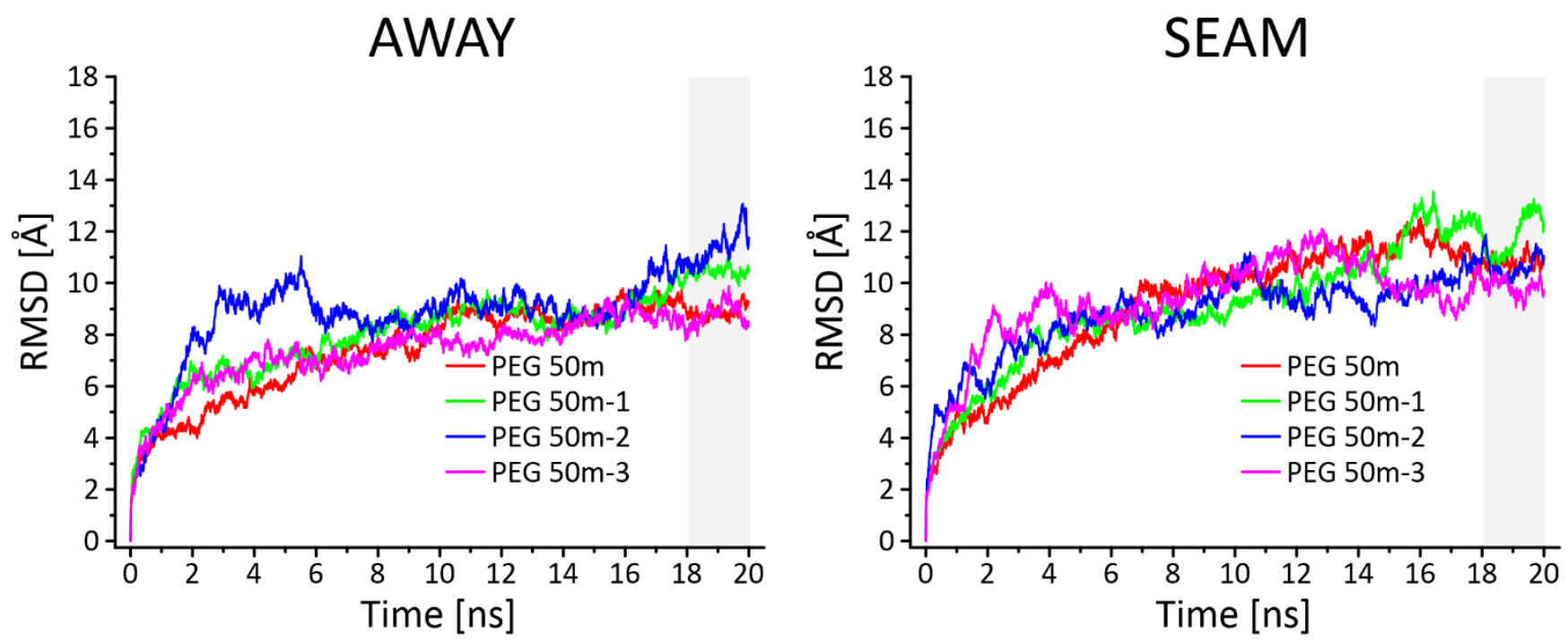

Figure S6 RMSD plots for all heavy atoms from PEG(50) and from each of the 3 PEG(50)R41 molecules from production runs of the AWAY (left) and the SEAM (right) systems with PEGs. Gray areas indicate the last 2 ns of the trajectories. 

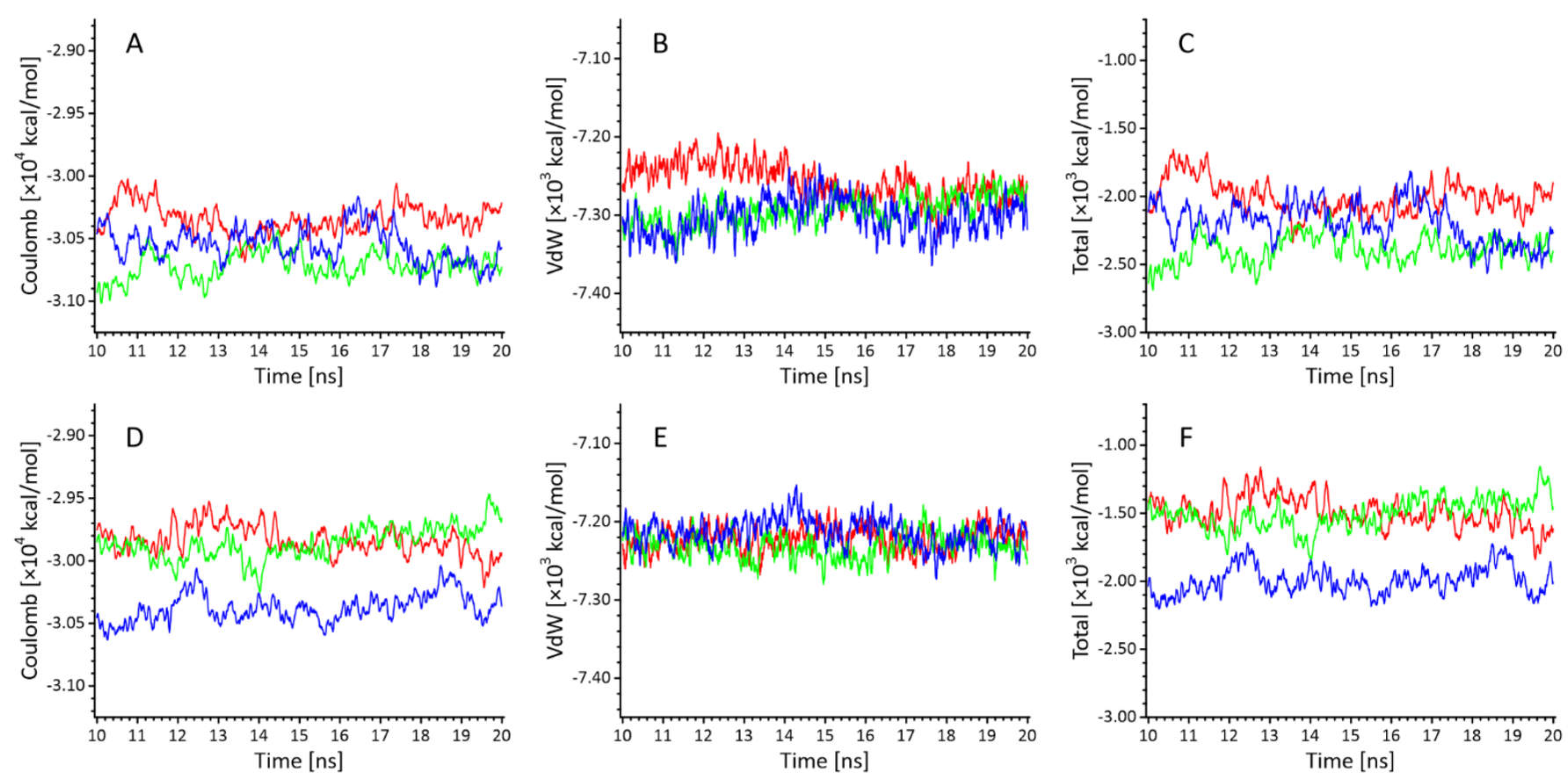

- MT

- MT+PEG(50) - MT+PEG(50)R41

Figure S7 Coulomb (A, D), van der Waals (B, E), and Total (C, F) energy plots for the last 10 ns of the production runs for the central dimers (see Figure S2) without PEGs (red), with PEG(50) (green), and with PEG(50)R41 (blue) molecules. These are moving average plots (for clarity, average from $+/-5$ points). Energies do not include water, ions, and the respective PEG molecule. Top row for the AWAY interface $(A, B, C)$, bottom row for the SEAM interface $(D, E$, F).
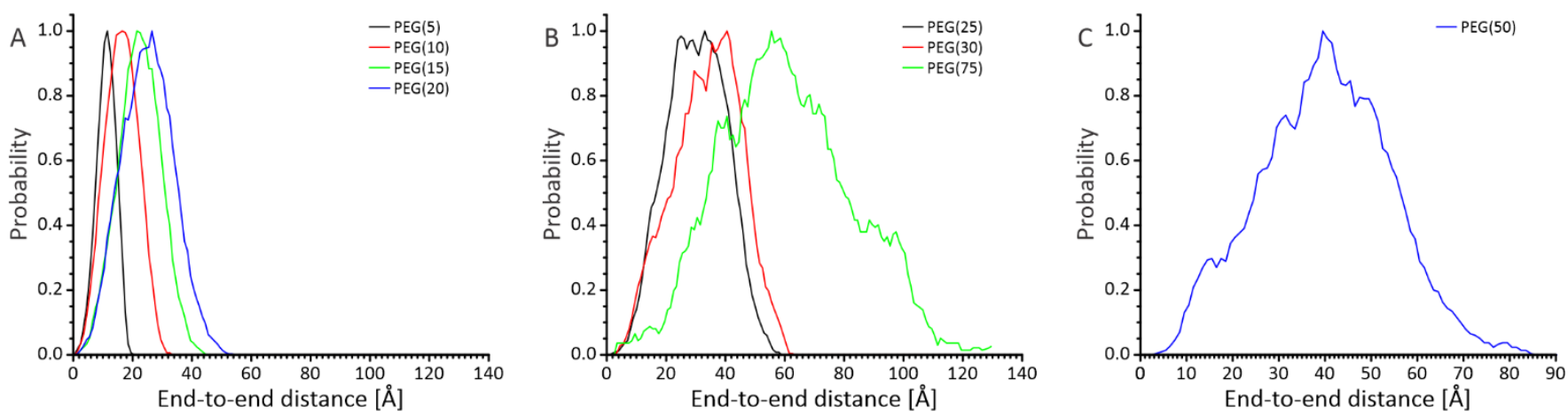

Figure $\mathbf{5 8}$ The normalized distribution of the end-to-end distances for PEG molecules calculated between terminal $\mathrm{O}$ atoms from $\mathrm{OH}$ groups from 1000 frames of our equilibration runs (5 ns). Panel A: PEG(5), PEG(10), PEG(15), and PEG(20); B: PEG(25), PEG(30), and PEG(75): C: PEG(50). 


\section{PEG(5) (200 Da)}
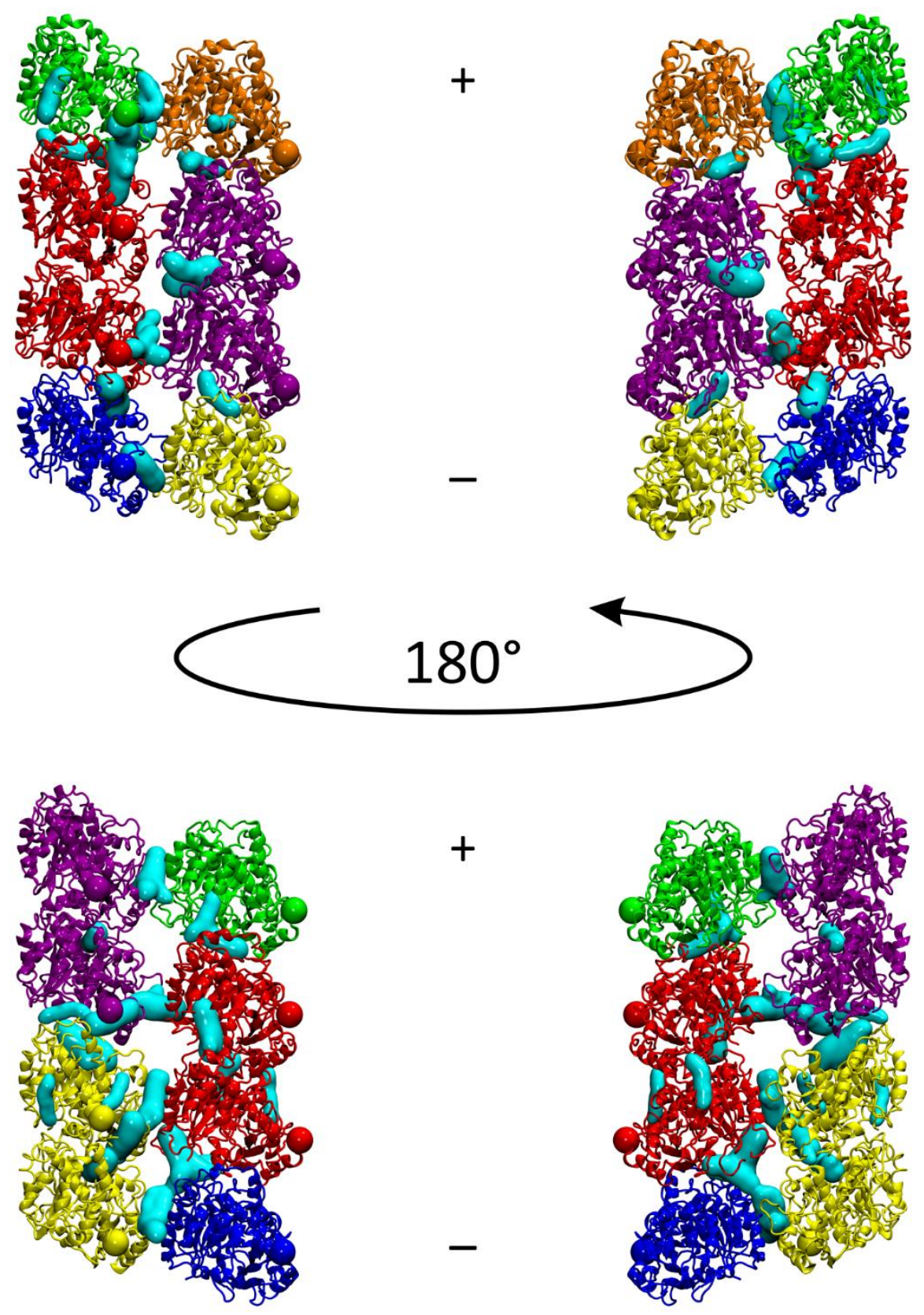

Figure S9A Docking of PEG(5), with the average mass of 200 Da or 5 monomers long, on the AWAY (top row) and SEAM (bottom row) interfaces (colors are the same as on Figure S1). The + and - ends of the MT are labeled. Cyan surfaces represent an overlap of 50 positions of PEG(5) with the lowest energy from each of three independent docking runs. Spheres represent the Cterminus amino acid of each monomer. Left panels - view from the outer surface of the MT, right panels - view from the inner surface (lumen) of the MT. 
$\operatorname{PEG}(10)(400 \mathrm{Da})$
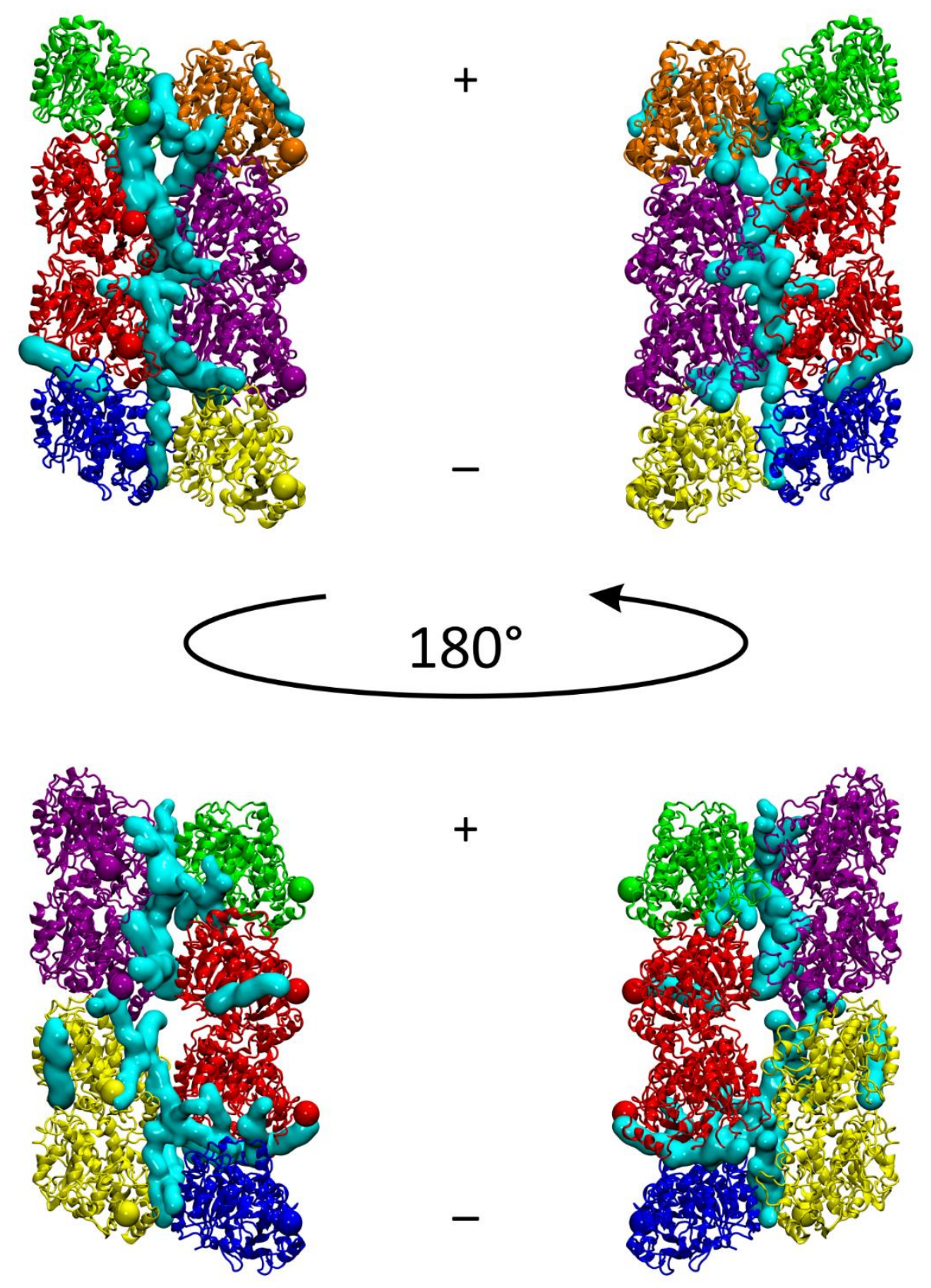

Figure S9B Same as Figure S9A for PEG(10), with the average mass of 400 Da or 10 monomers long. 
$\operatorname{PEG}(15)(600 \mathrm{Da})$
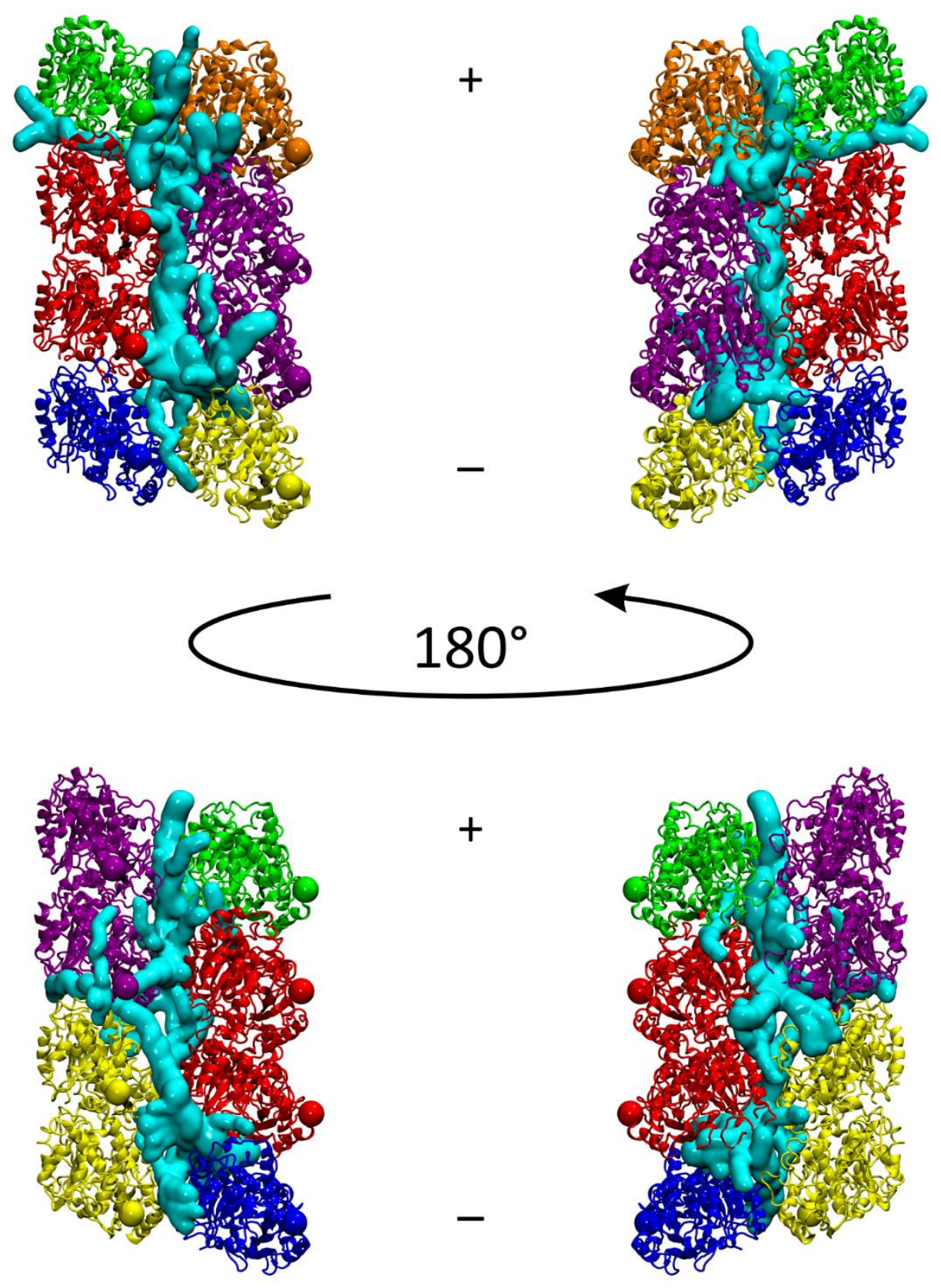

Figure S9C Same as Figure S9A for PEG(15), with the average mass of 600 Da or 15 monomers long. 
PEG(20) (800 Da)
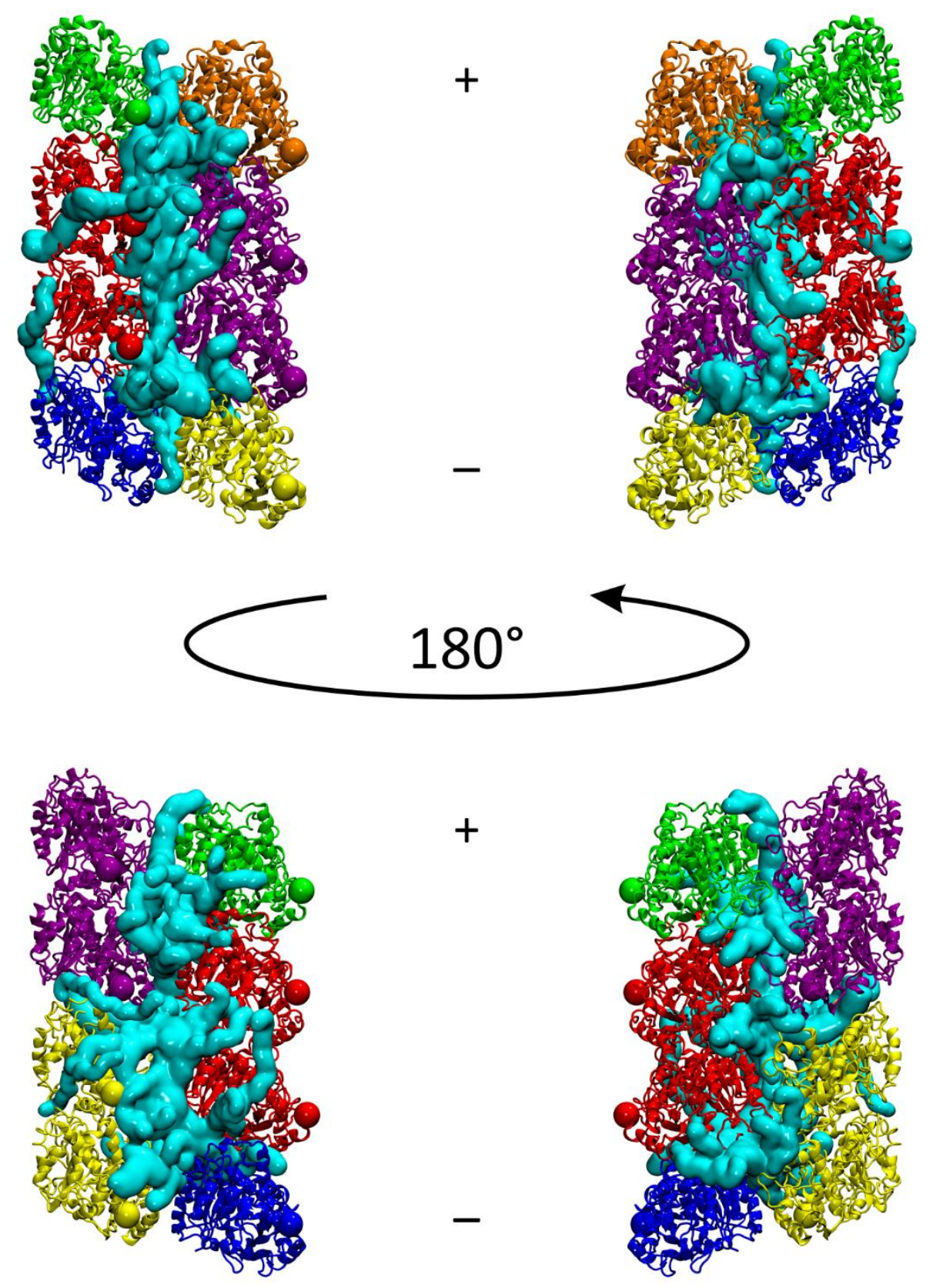

Figure S9D Same as Figure S9A for PEG(20), with the average mass of 800 Da or 20 monomers long. 
PEG(25) (1000 Da)
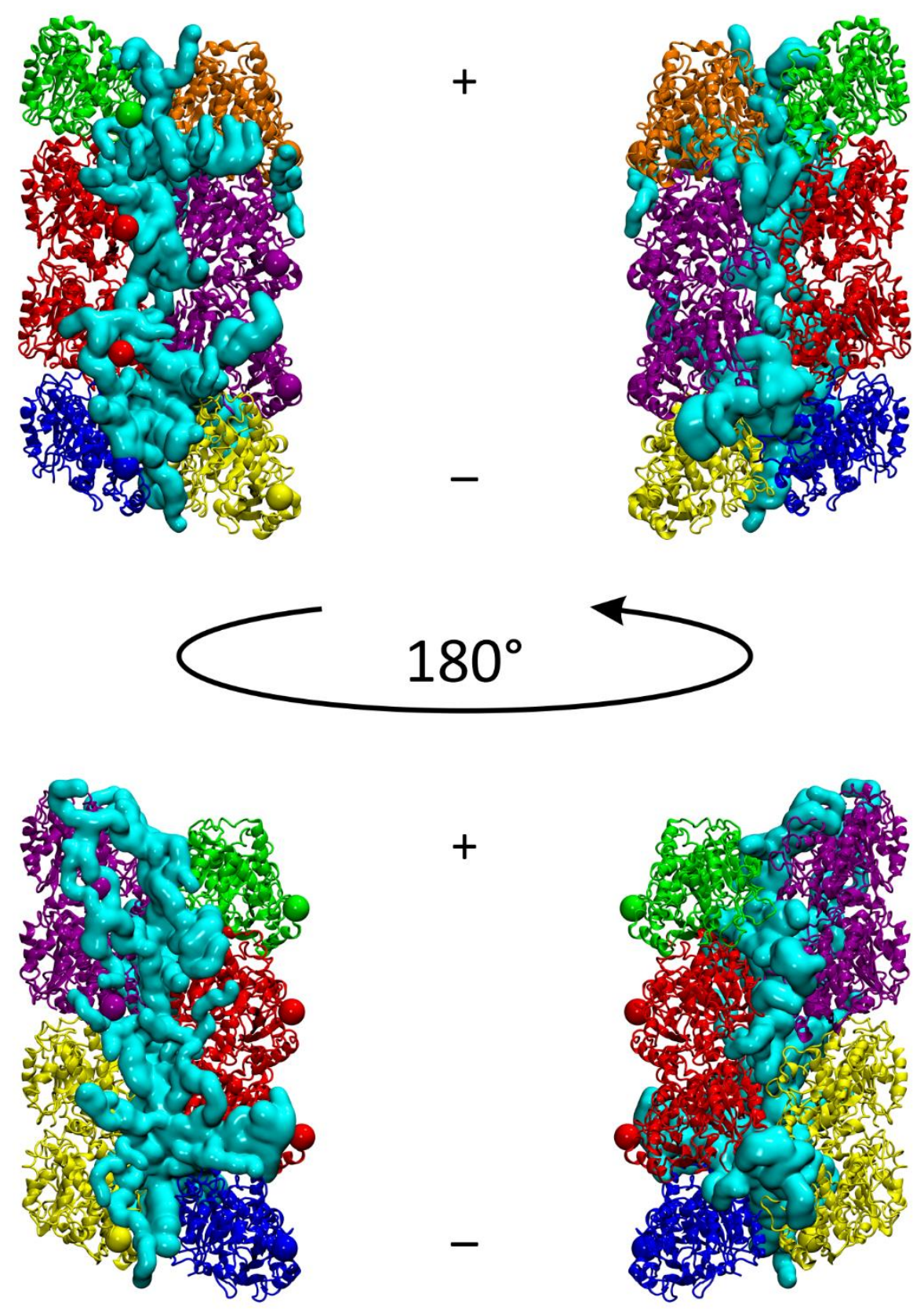

Figure S9E Same as Figure S9A for PEG(25), with the average mass of 1000 Da or 25 monomers long. 
PEG(30) (1200 Da)
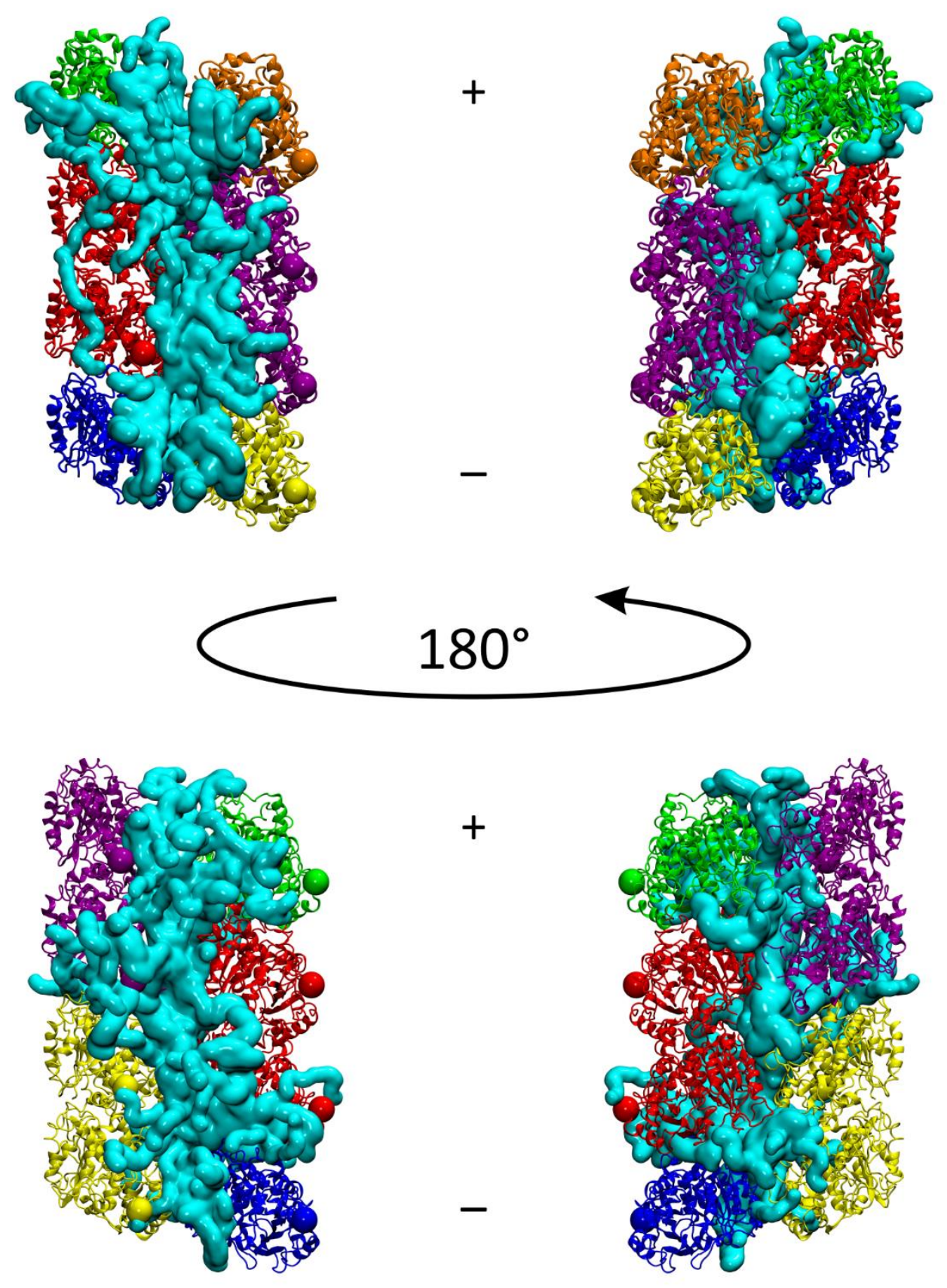

Figure S9F Same as Figure S9A for PEG(30), with the average mass of 1200 Da or 30 monomers long. 
PEG(35) (1400 Da)
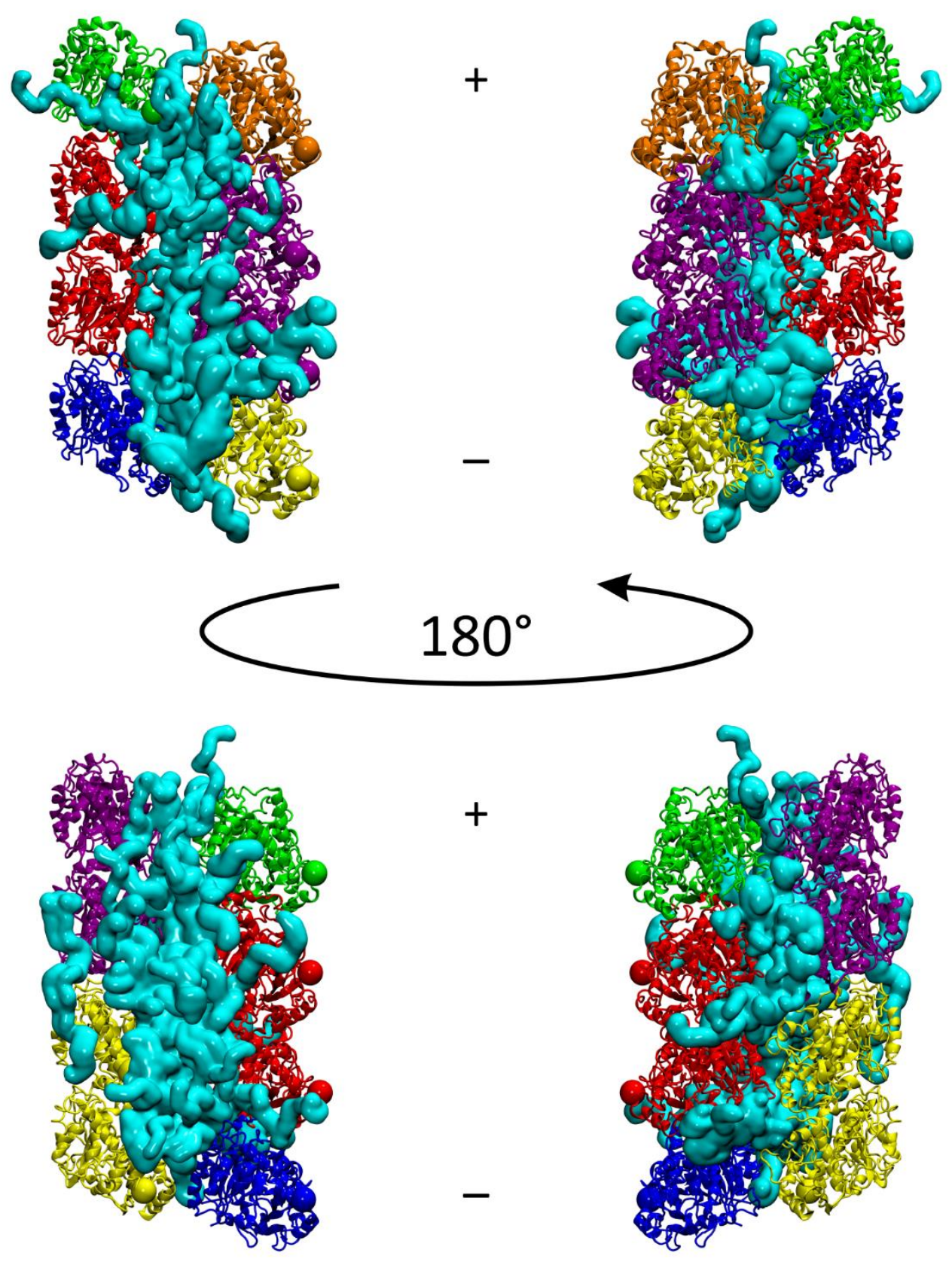

Figure S9G Same as Figure S9A for PEG(35), with the average mass of 1400 Da or 35 monomers long. 
PEG(40) (1600 Da)
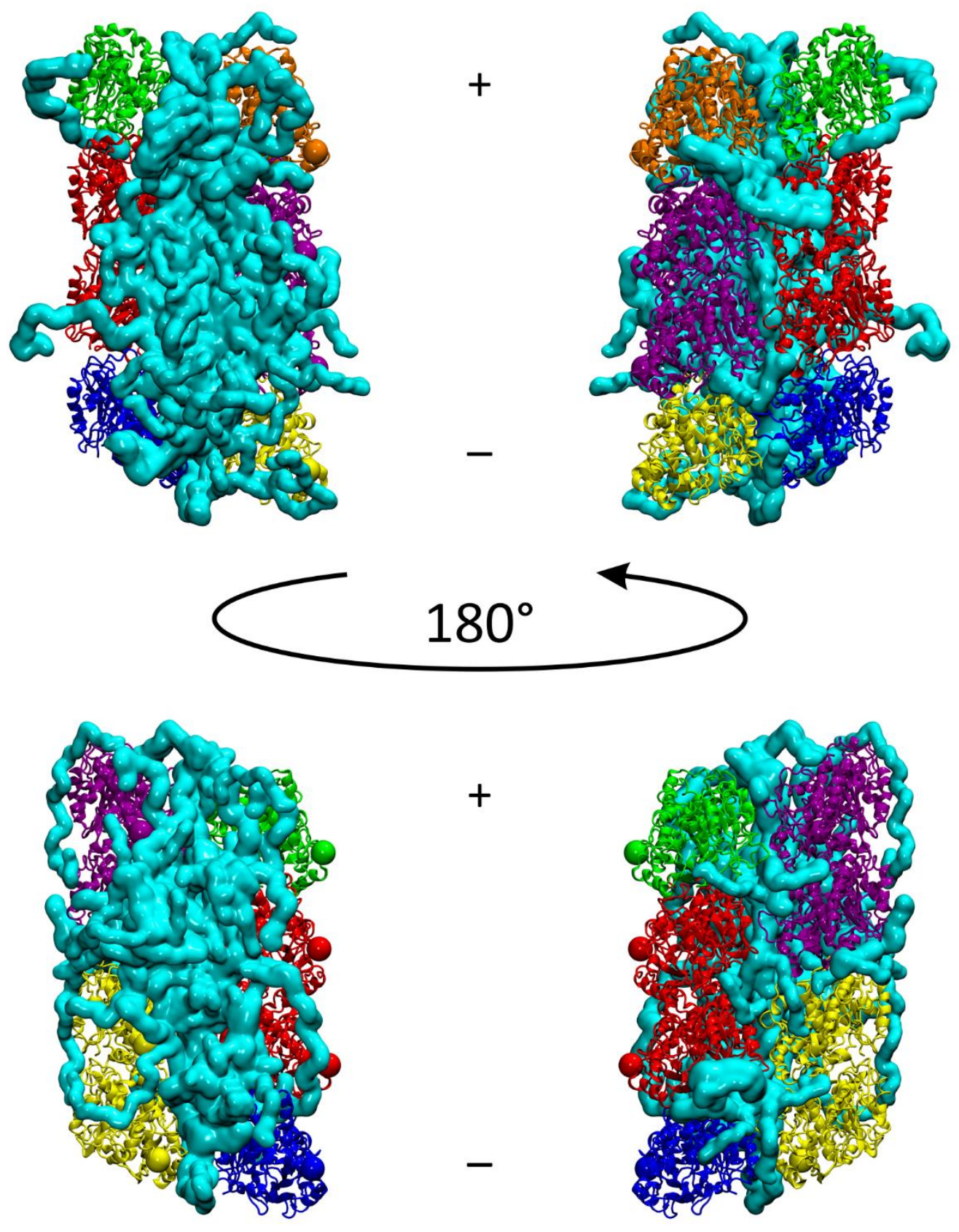

Figure S9H Same as Figure S9A for PEG(40), with the average mass of 1600 Da or 40 monomers long. 
PEG(45) (1800 Da)
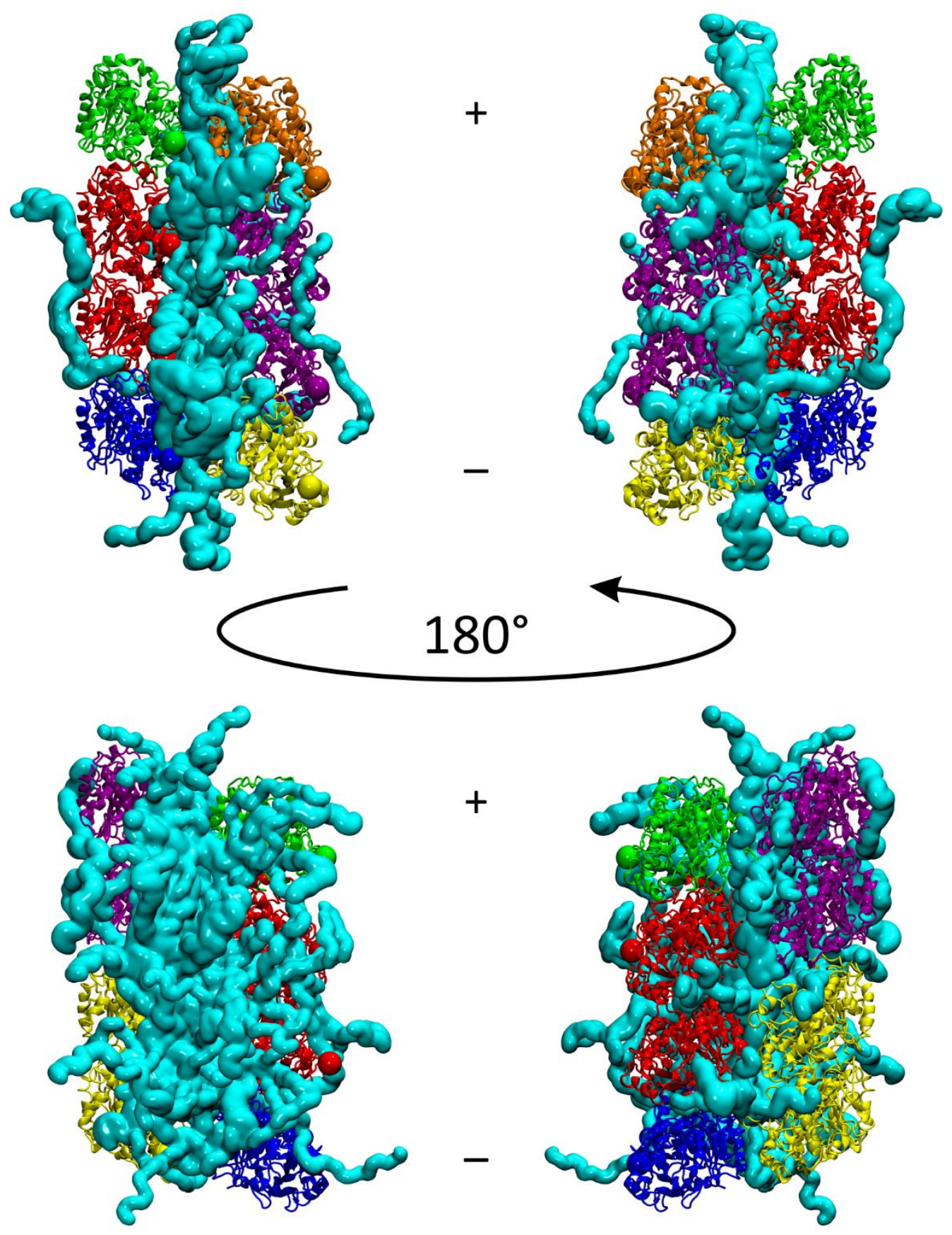

Figure S9I Same as Figure S9A for PEG(45), with the average mass of 1800 Da or 45 monomers long. 


\section{PEG(55) (2200 Da)}
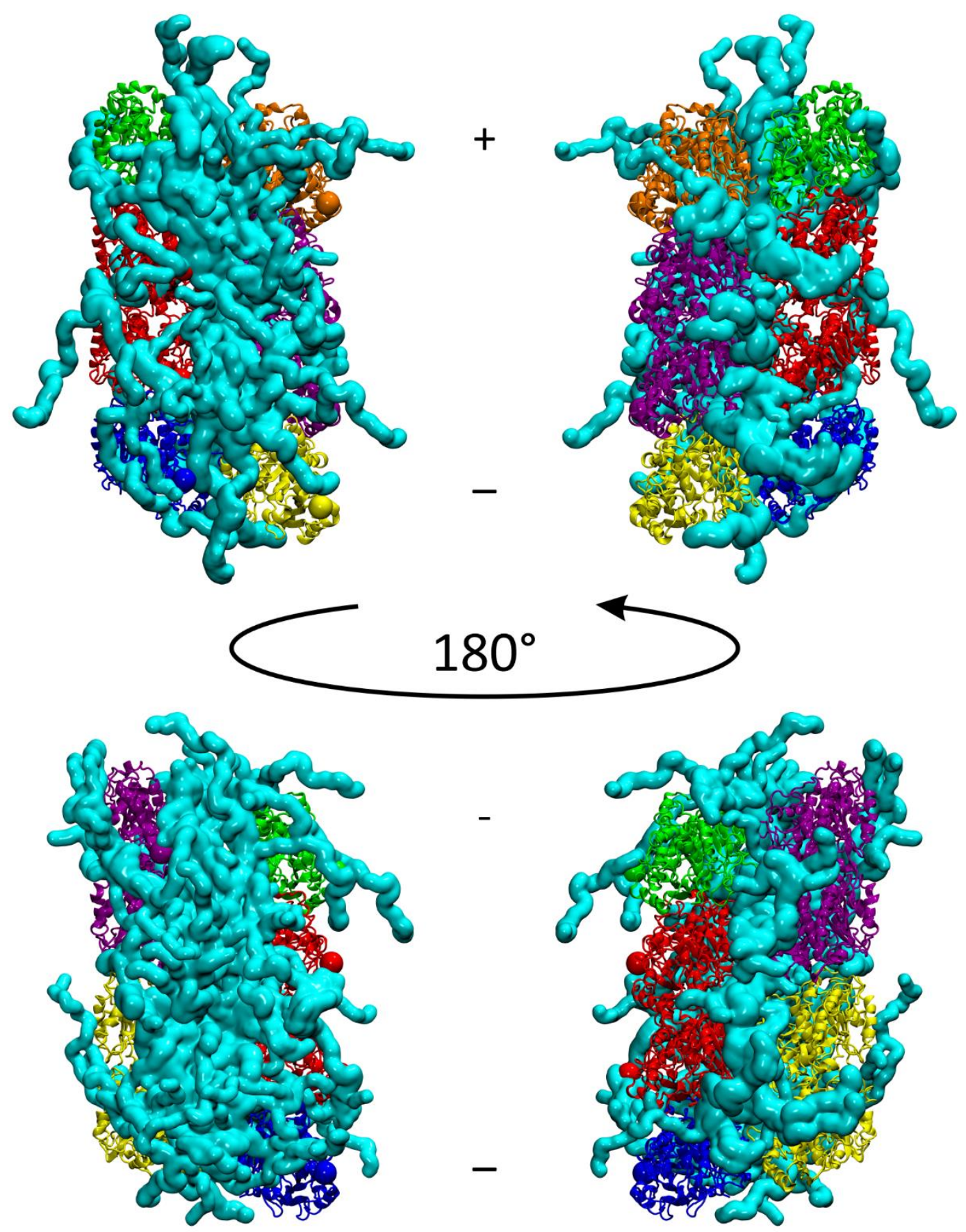

Figure S9J Same as Figure S9A for PEG(55), with the average mass of 2200 Da or 55 monomers long. 


$$
\text { PEG(60) (2400 Da) }
$$

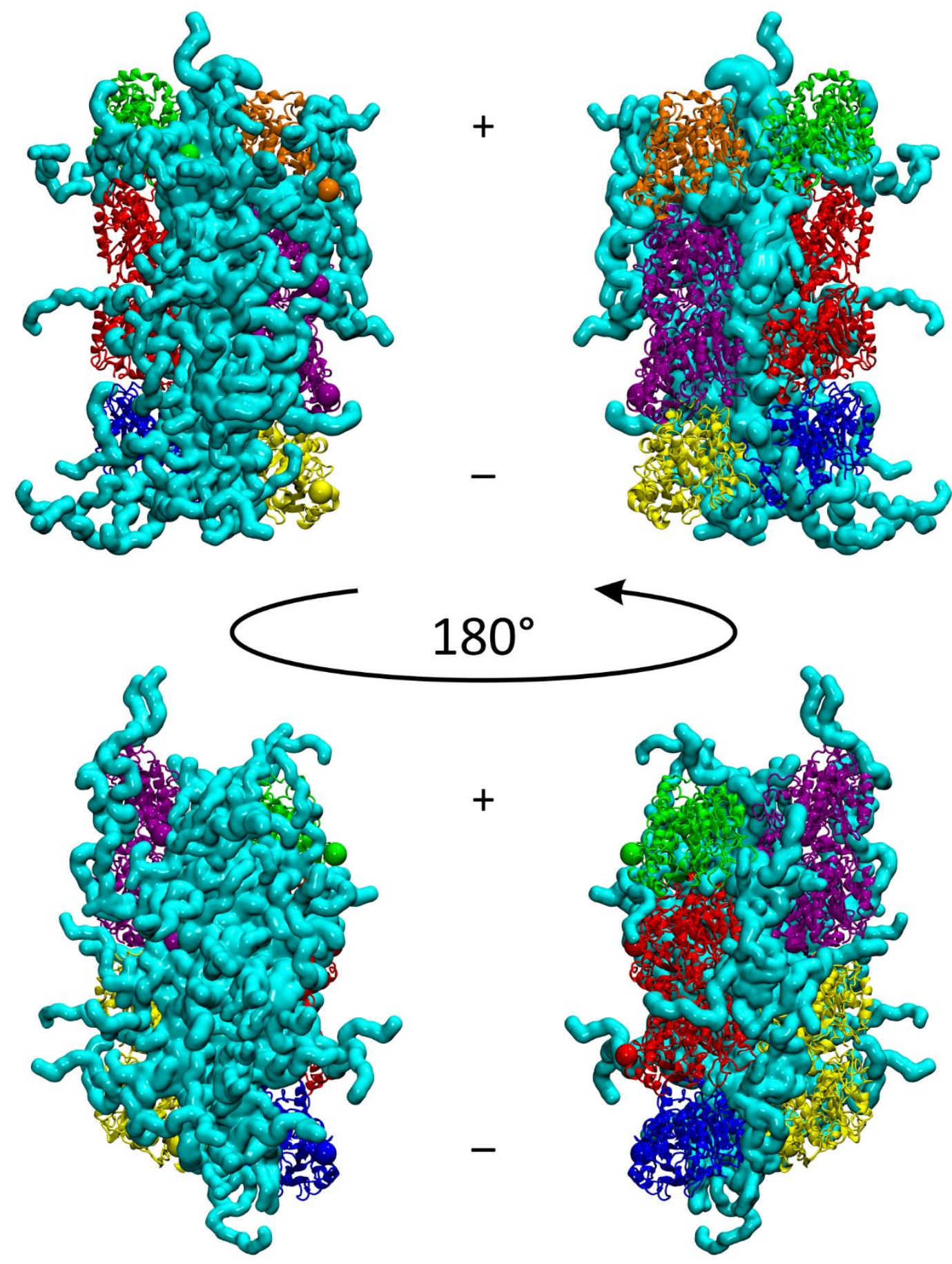

Figure S9K Same as Figure S9A for PEG(60), with the average mass of 2400 Da or 60 monomers long. 


$$
\text { PEG(65) (2600 Da) }
$$
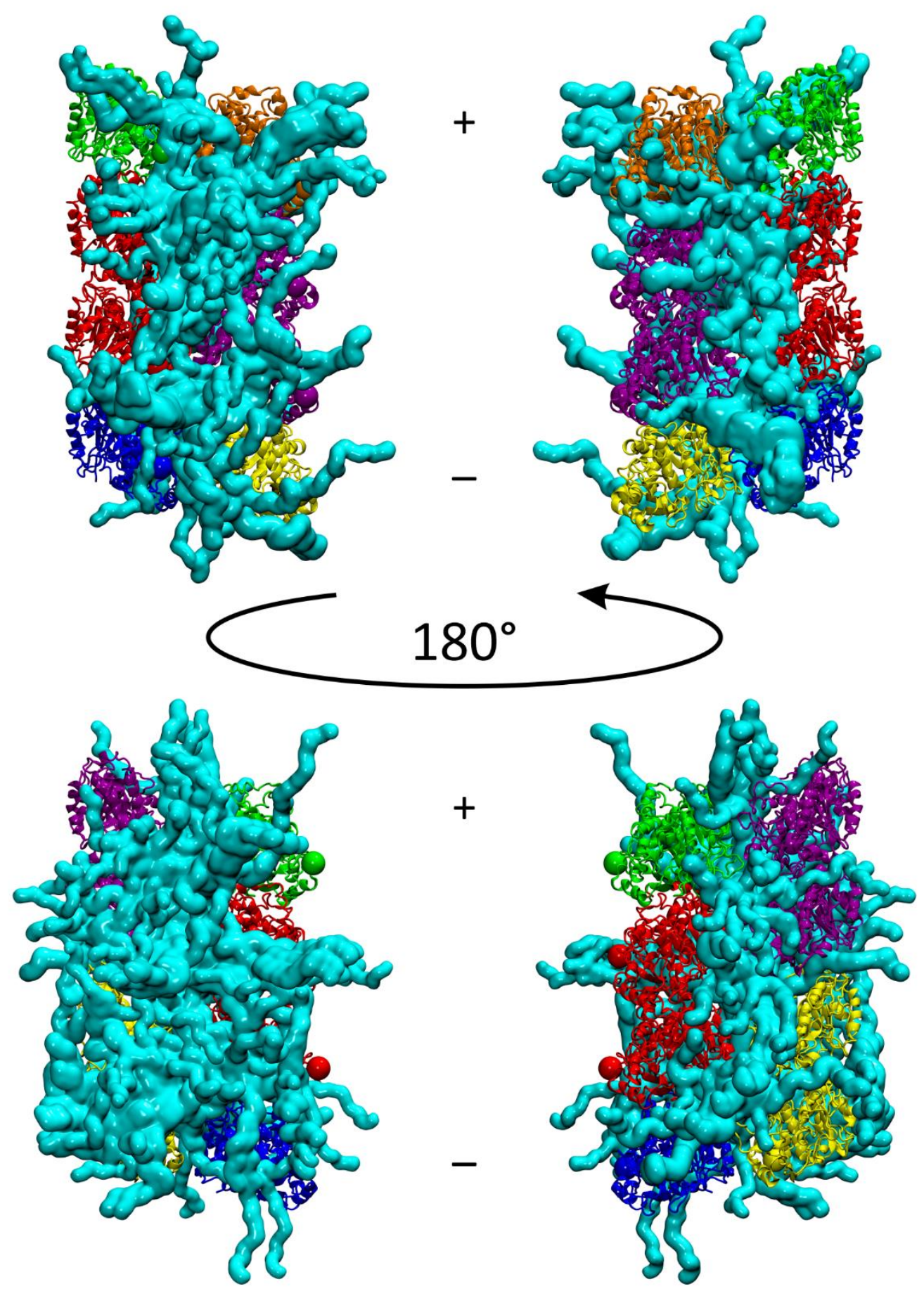

Figure S9L Same as Figure S9A for PEG(65), with the average mass of 2600 Da or 65 monomers long. 


\section{PEG(70) (2800 Da)}
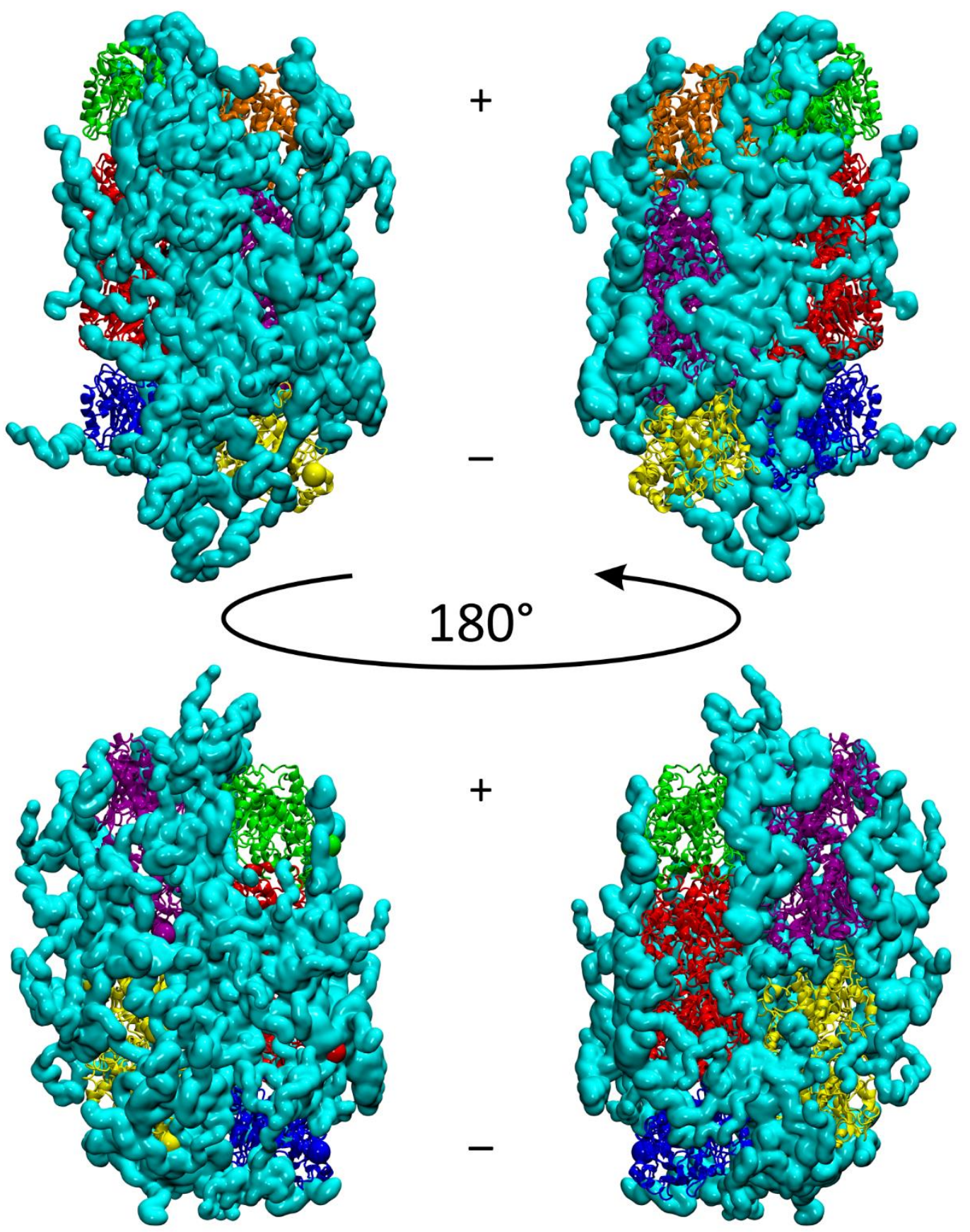

Figure S9M Same as Figure S9A for PEG(70), with the average mass of 2800 Da or 70 monomers long. 


\section{PEG(75) (3000 Da)}
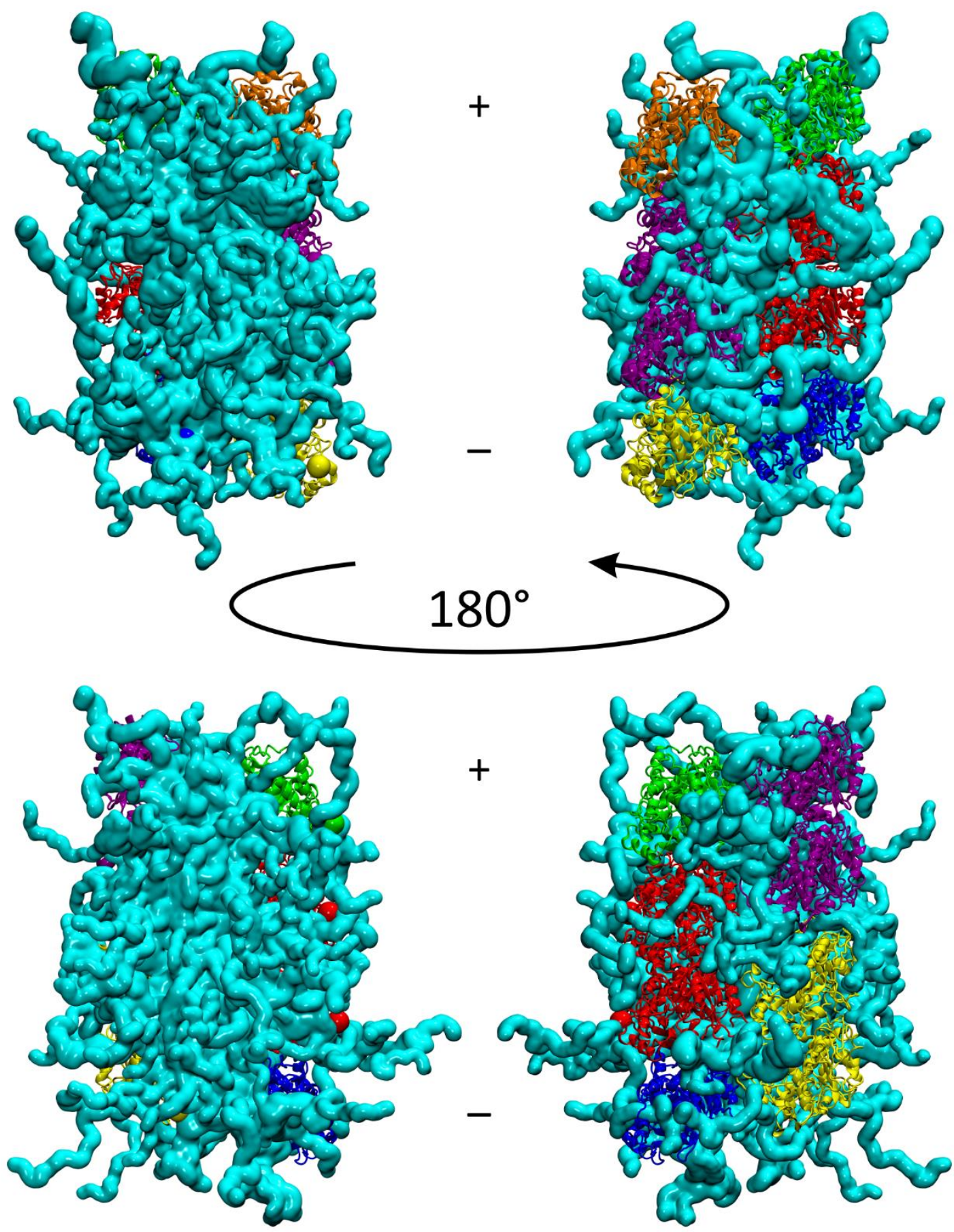

Figure S9N Same as Figure S9A for PEG(75), with the average mass of 3000 Da or 75 monomers long. 


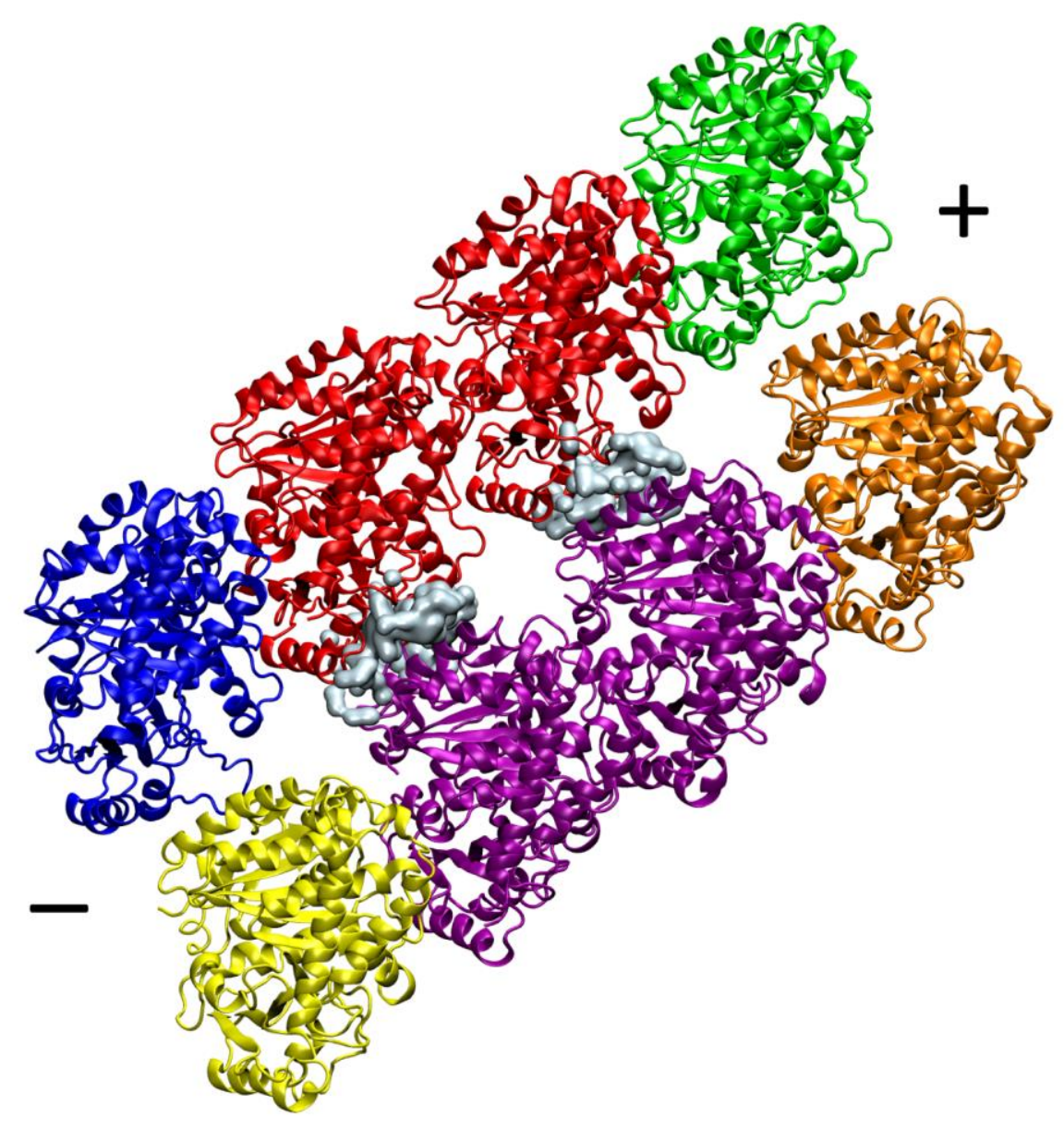

Figure S10 Example of water molecules located within $3.5 \AA$ from the central dimer (red) and at the same time within $3.5 \AA$ from its adjacent tubulin monomers displayed as a bluish surface for the last frame of the production run for the AWAY model without PEG(50). The + and - ends of the MT are indicated. Tubulin monomers colors follow Figure S1. 
A

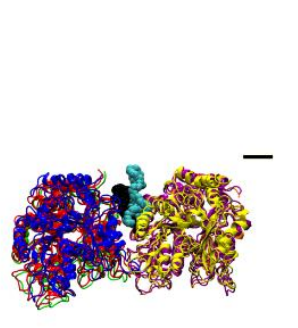

D

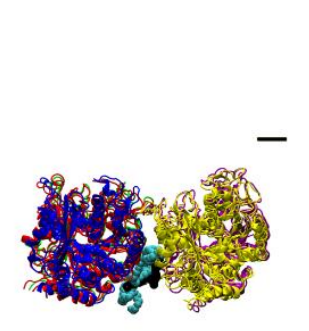

B

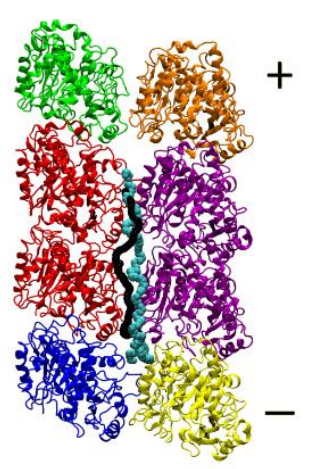

$\mathbf{E}$

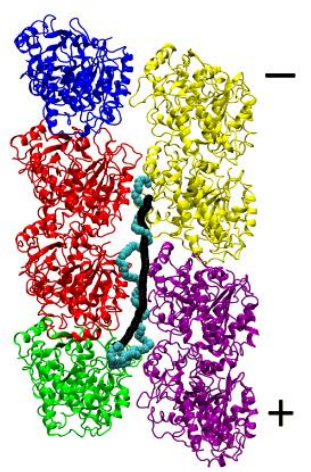

C

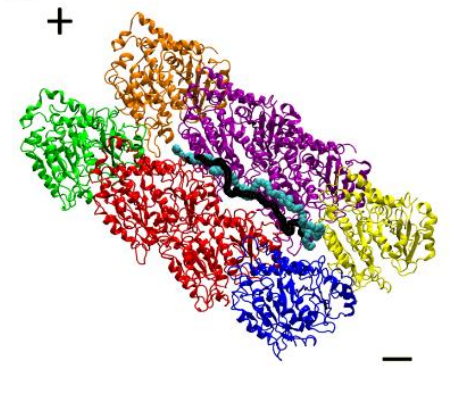

$\mathbf{F}$

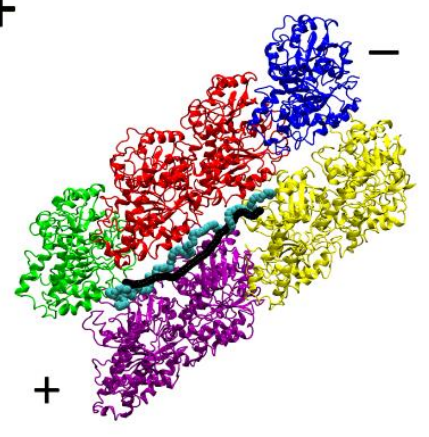

Figure S11A Comparison of the initial position of the PEG(50) molecule (cyan spheres) in our full atomistic MD studies vs the position of PEG(50) (black) in the average structure from the last 2 ns of production runs. View from the + end (A - AWAY, D - SEAM), from the top (B AWAY, E - SEAM), and from an angle (C - AWAY, F - SEAM). Full dimers have the same colors; the + and - ends of the MT fragments are indicated.

A

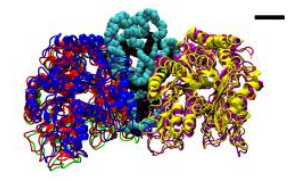

B

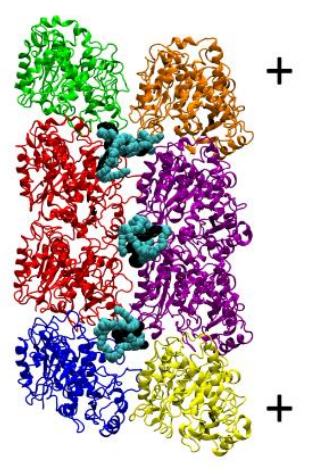

D

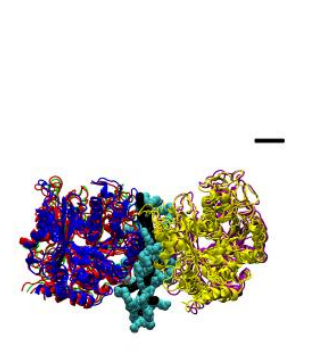

$\mathbf{E}$

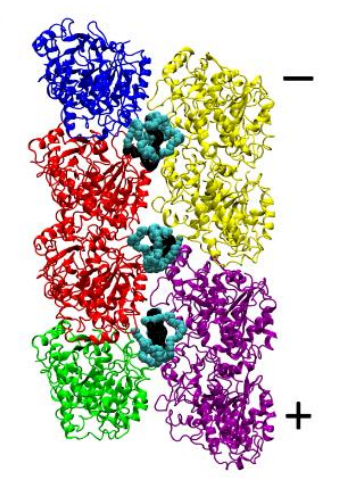

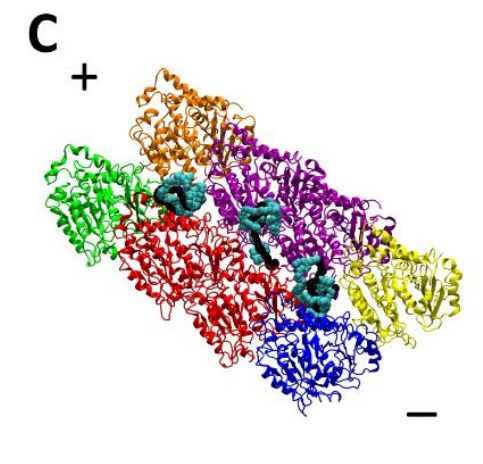

$\mathbf{F}$

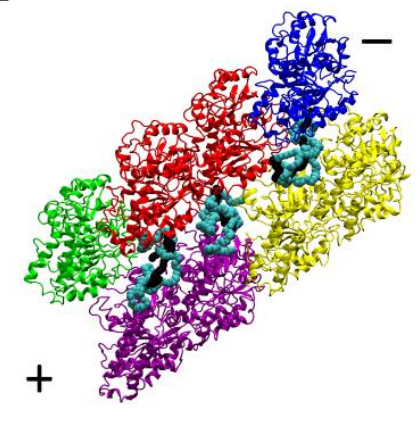

Figure S11B Similar to Figure S11a for the PEG(50)R41 molecule. 


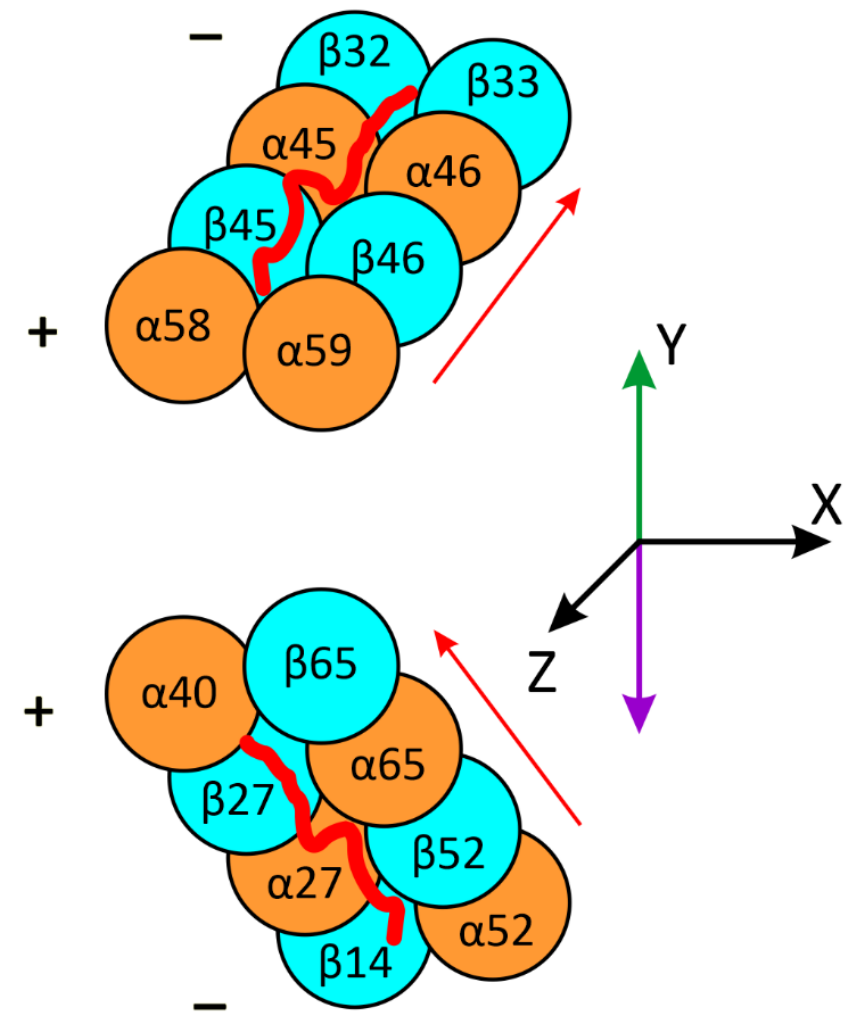

Figure S12 Schematics of the PEG(50) position analysis indicating the direction of the shift. Top structure is for the AWAY+PEG(50) model, and the bottom is for the SEAM+PEG(50). The colors of the tubulin monomers and the name codes are the same as in Figure S11. The plus and minus ends are indicated. PEG(50) is shown as a red curve on the top of the MT surface. Red arrows indicate the direction of the PEG(50) atoms ( $O$ from 1 to 151, see Table S5). Notice the opposite orientation of PEG(50) in the AWAY versus the SEAM models. The $X$ and $Z$ axes directions are the same for both models. The arrows on the $Y$-axis indicate the displacement directions away from the MT surface: green for the AWAY+PEG(50) model and magenta for the SEAM+PEG(50) model. 

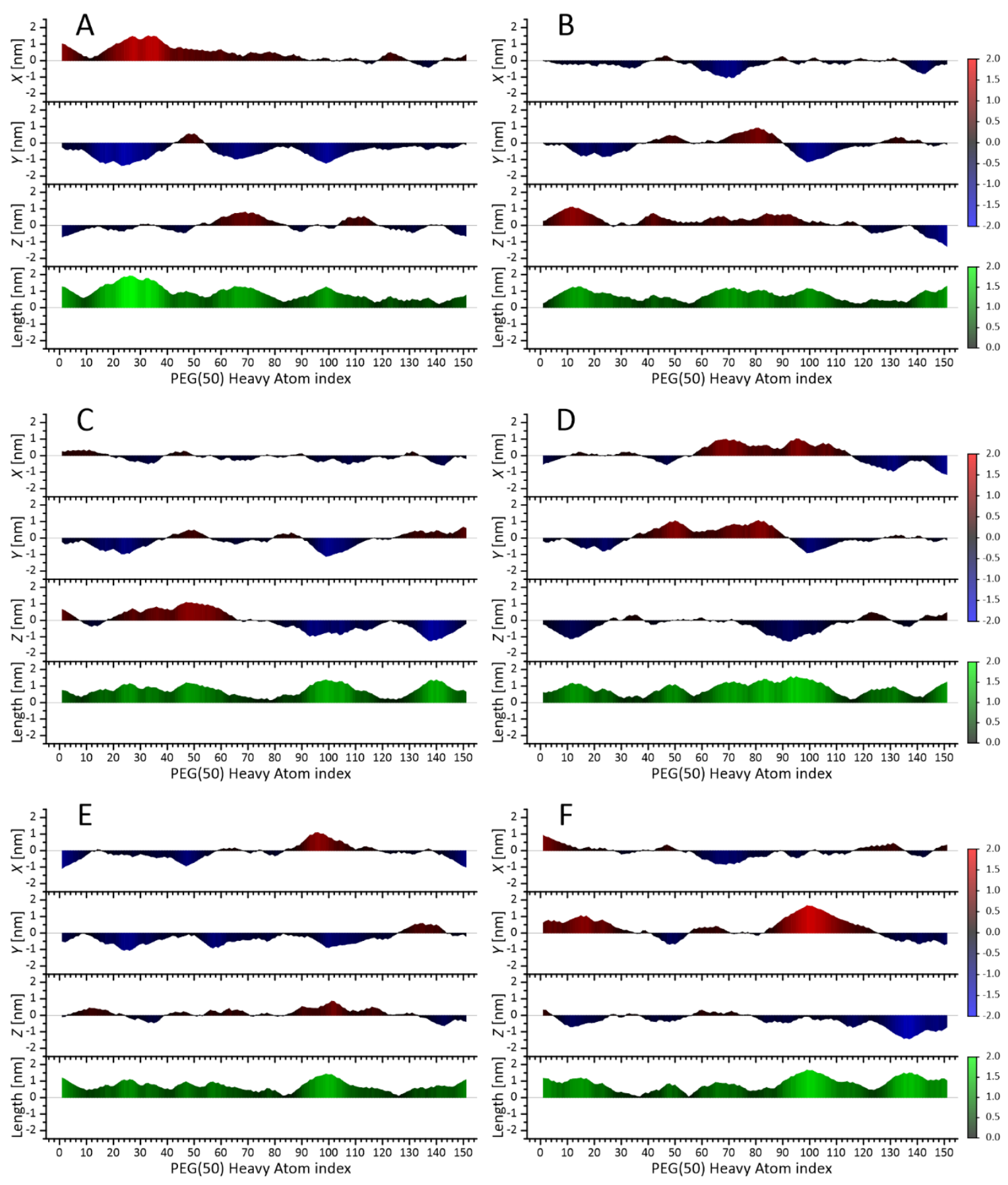

Figure S13 Change in the position of the heavy atoms of the three PEG(50)R41 molecules in the AWAY+PEG(50)R41 model (panel A, C, D), and the SEAM+PEG(50)R41 model (panel B) calculated as the difference between the position in the average structure (from the last $2 \mathrm{~ns}$ of the production run) and the initial PEG(50)R41 positions after docking. Panels from top to bottom: displacement on the X-axis, Y-axis, Z-axis, and the amplitude of the displacement. Gradient color codes are in the right panel. A positive change in the displacement on the X-axis indicates a shift of the position of an atom away from the central dimer (toward the adjacent tubulin monomers: $\mathrm{a} 46$ and $\beta 46$ for AWAY+PEG(50)R41, and $\beta 52$ and $\mathrm{a} 65$ for SEAM+PEG(50)R41; see Figure S2), a positive value of the $Y$-axis displacement indicates a shift away from the MTs surface, and a positive displacement on the Z-axis indicates a shift toward the + end of the MT (see Figure S12). The PEG(50)R41 heavy atom index is in Table S5. 

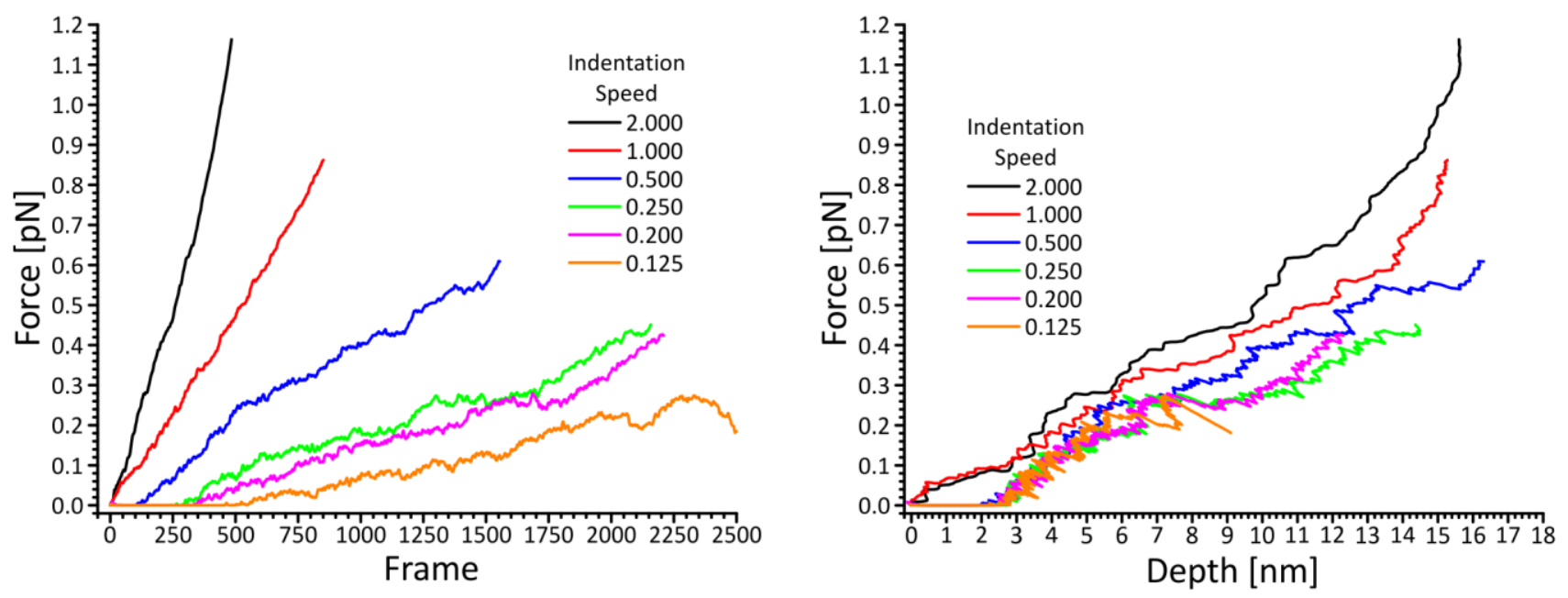

Figure S14 An example of Force vs frame (left) and Force vs depth (right) plots for single trajectories of 13 MTx8 P1/P13 (SEAM) compression using the BT set-up at various indentation speeds.

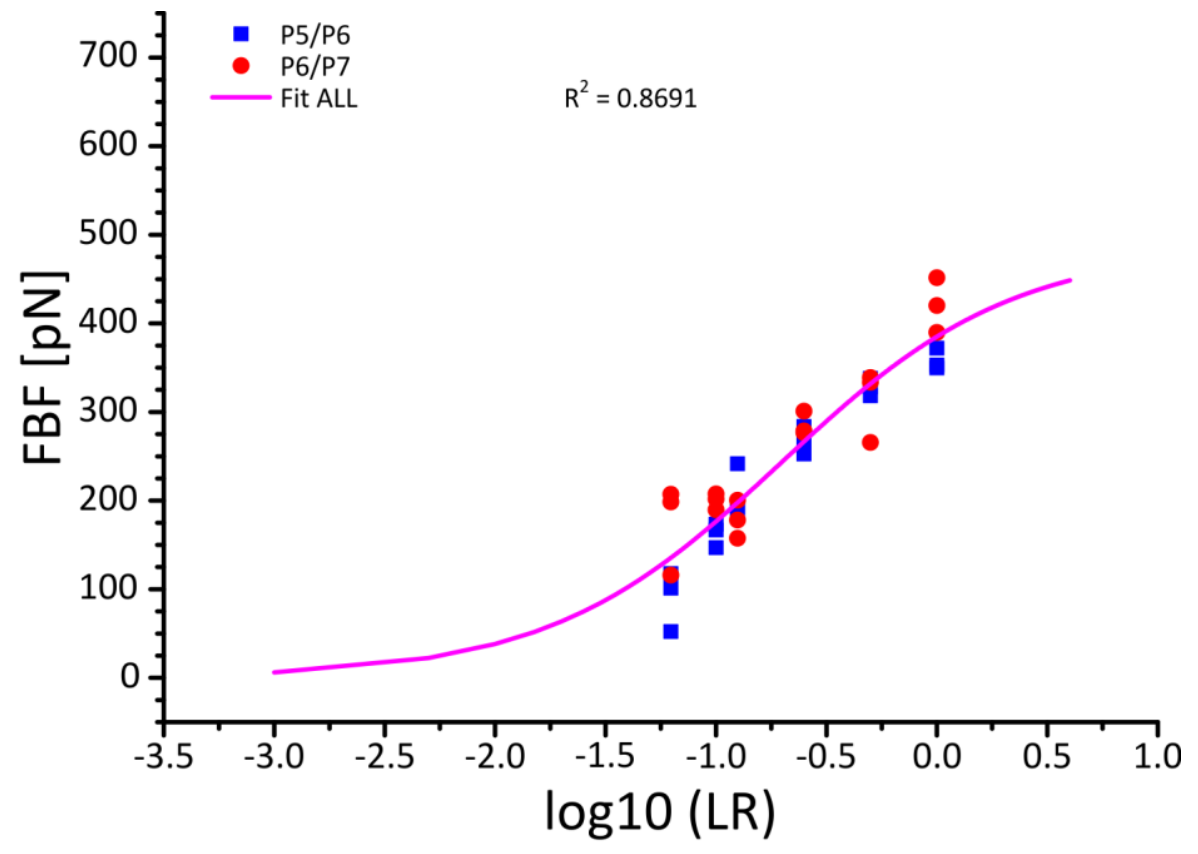

Figure S15 FBFs vs. log of the relative loading rate (LR) for the ST models of indentation at positions P5/P6 and P6/P7. The fitting by a sigmoidal function for all data points is shown (Fit ALL). 


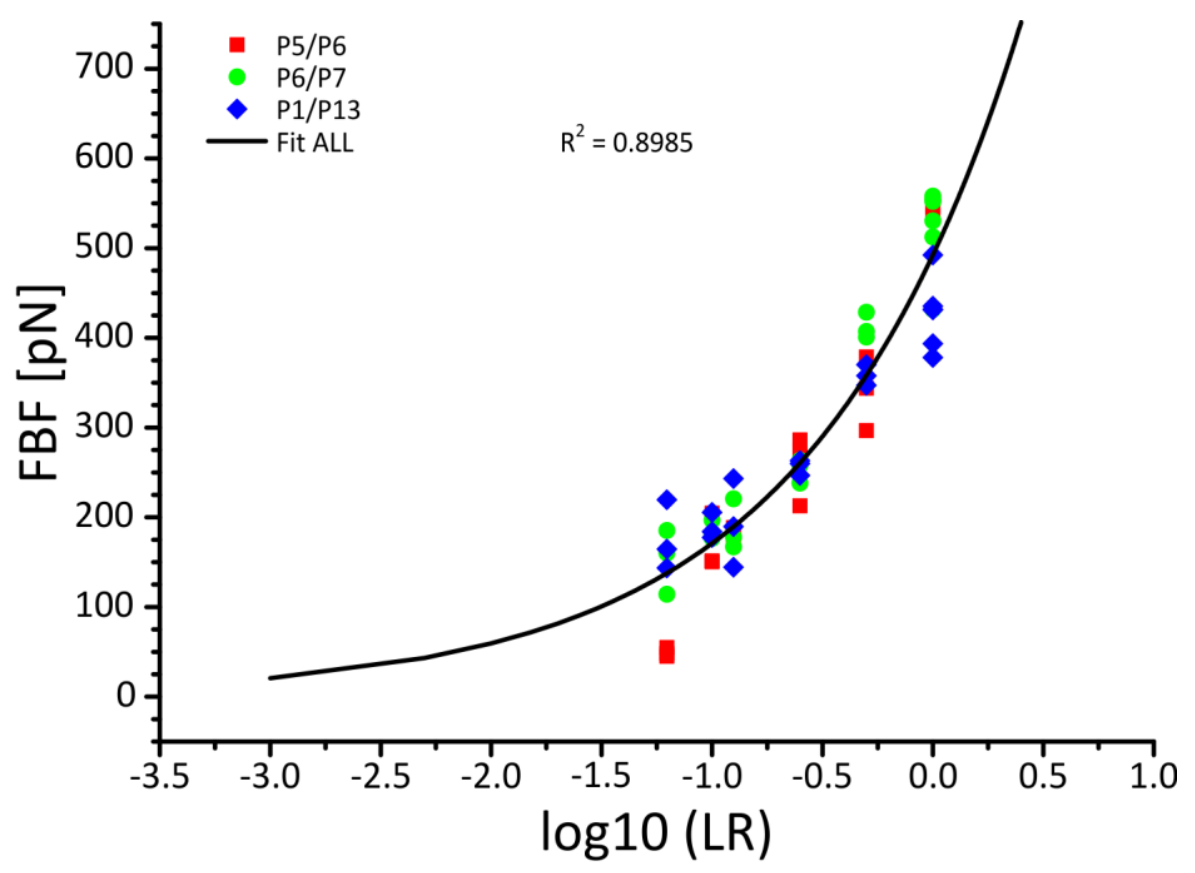

Figure S16 FBFs vs. log of the relative loading rate (LR) for BT models of compression at positions P5/P6, P6/P7, and P1/P13. The fitting by an exponential function for all data points is shown (Fit ALL).

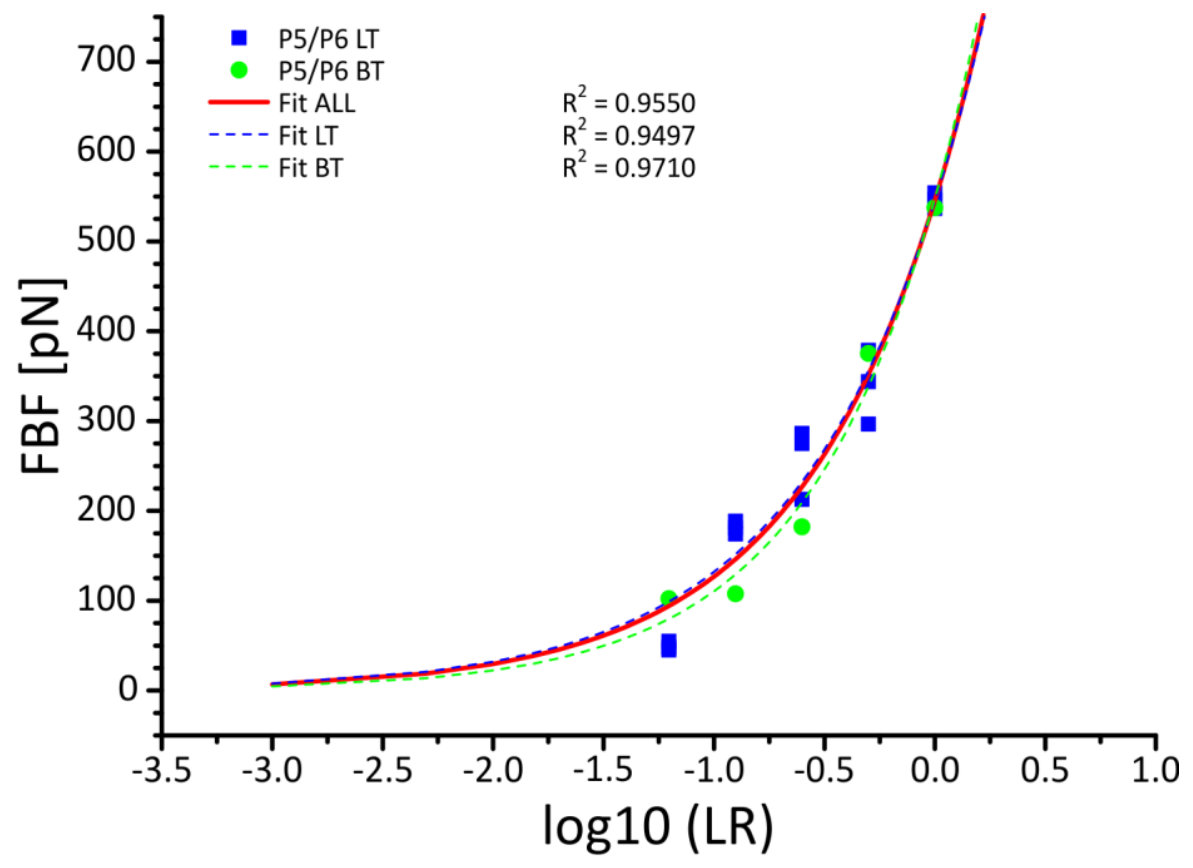

Figure S17 FBFs vs. log of the relative loading rate (LR) for the 6 dimers long MT models of compression at positions P5/P6 with LT (P5/P6 LT) and BT (P5/P6 BT). The fitting exponential functions for all data points (Fit ALL), LT only (Fit LT), and BT only (Fit BT) are also shown. 


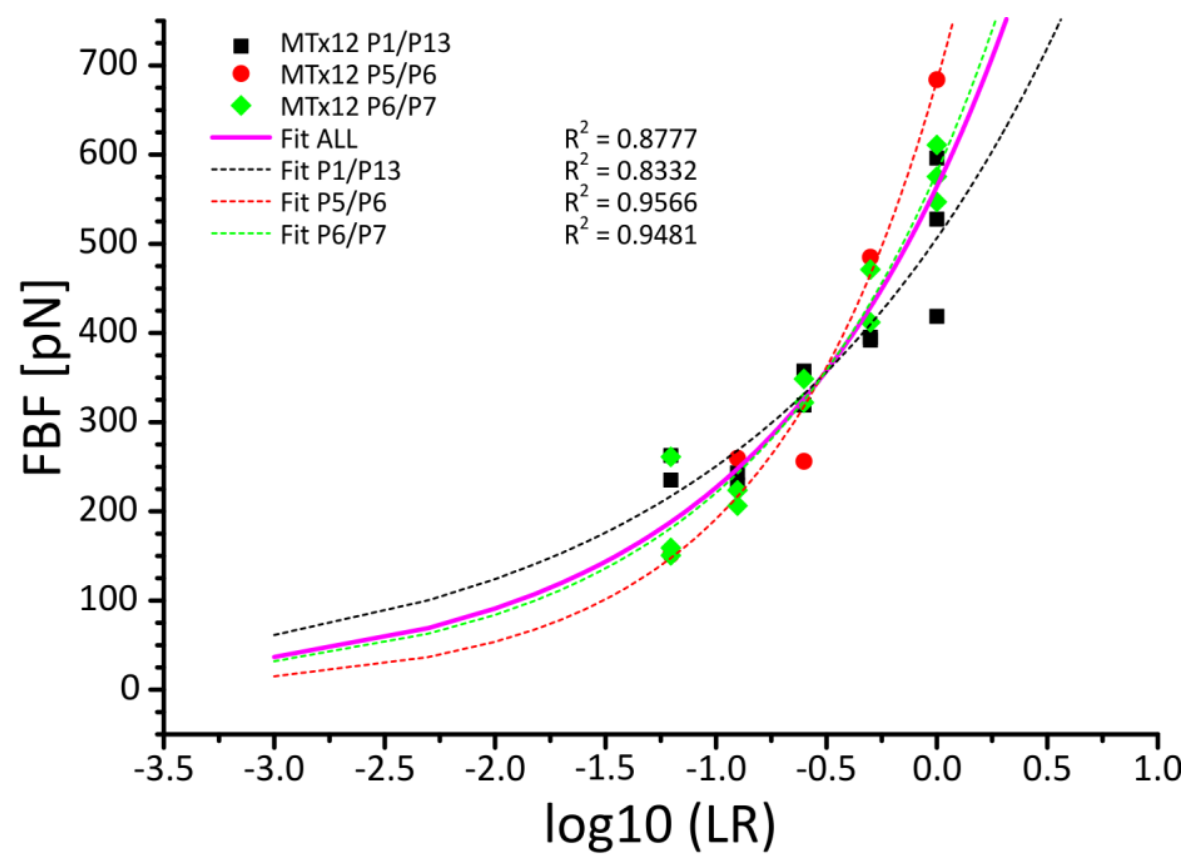

Figure S18 FBFs vs. log of the relative loading rate (LR) for the 12 dimers long MT models with BT of compression at positions P1/P13 (MTx12 P1/P13), position P5/P6 (MTx12 P5/P6), and position P6/P7 (MTx12 P6/P7). The fitting exponential functions for all data points (Fit ALL), only for position P1/P13 (Fit P1/P13), only for position P5/P6 (Fit P5/P6), and only for position P6/P7 (Fit P6/P7) are also shown.
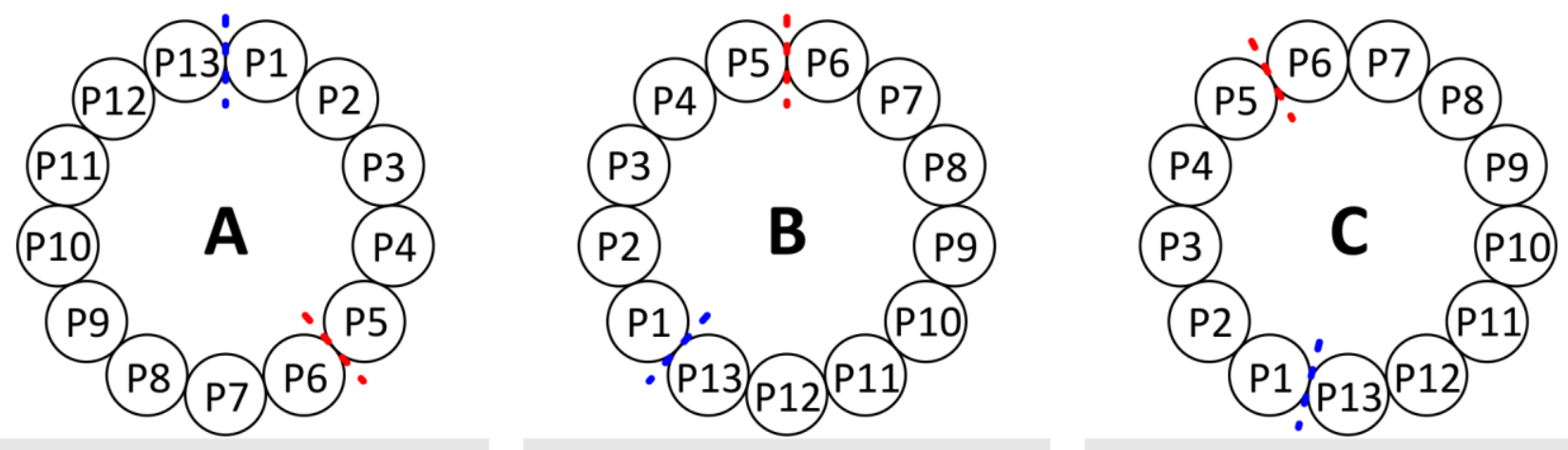

Figure S19 Schematics showing the relative position of the SEAM (P1/P13, blue) interface and of the weakest lateral interface in the MT P5/P6 (red) above the plate (gray bar) for indentation between P1/P13 (A, the SEAM), P5/P6 (B), and P6/P7(C). The view is from the - end of the MT filament. 

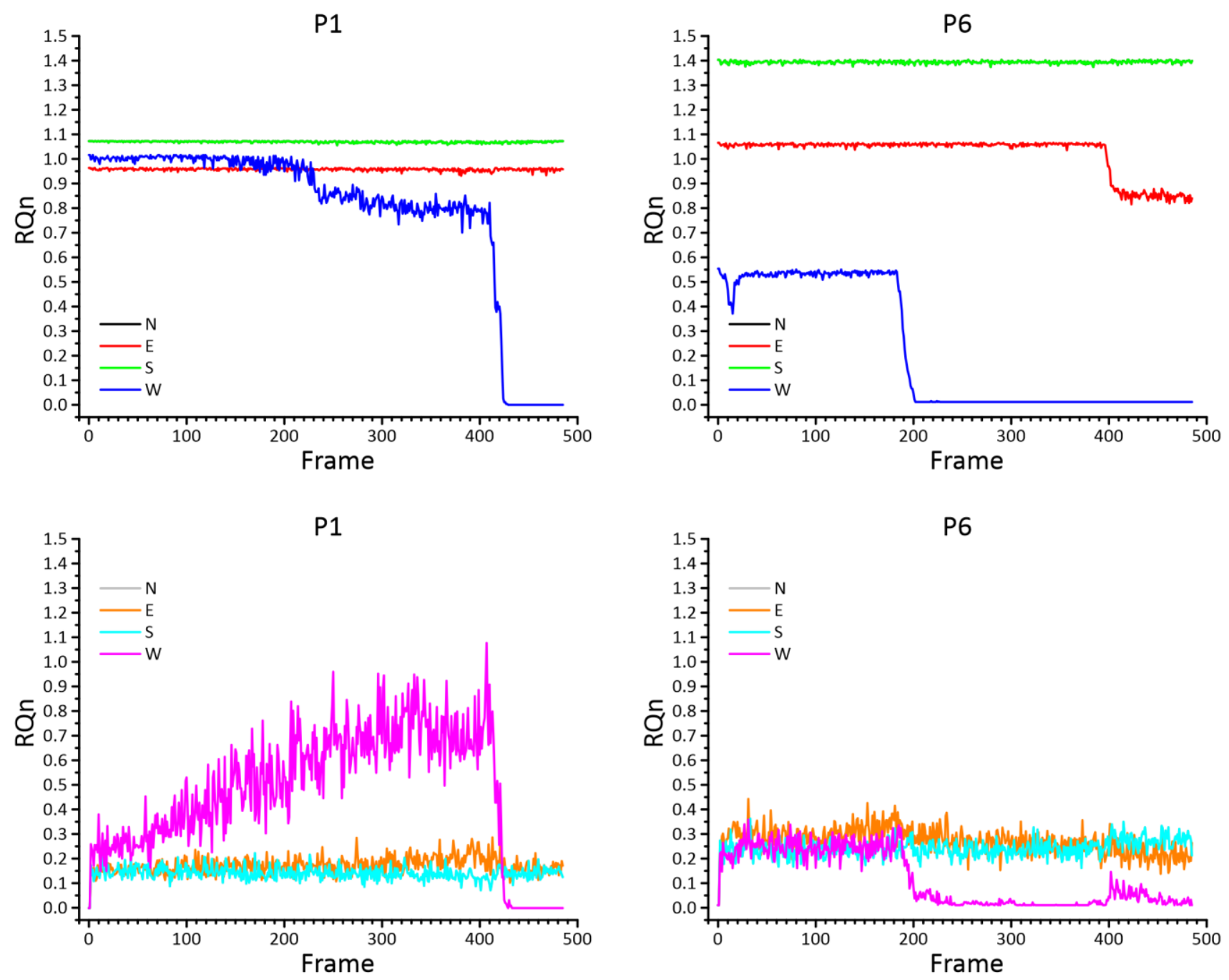

Figure S20 An example of RQn plots for 13MTx8 P1/P13 compression using the BT set-up at $2.0 \mu \mathrm{m} / \mathrm{s}$. Top row - native contacts, bottom row - non-native contacts. Left hand side: plot for protofilament 1 (P1): N - longitudinal contacts toward + end on $\mathrm{P} 1 ; \mathrm{S}$ - longitudinal contacts toward - end on P1; E lateral contacts between P1 and P2; W - lateral contacts between P1 and P13. Right hand side: plot for protofilament 6 (P6): $N$ - longitudinal contacts toward + end on P6; S - longitudinal contacts toward - end on P6; E lateral contacts between P6 and P7; W lateral contacts between P61 and P5. Rapid drops of the RQn values for native and non-native contacts to 0 for $W(P 1)$ and $W(P 6)$ indicate a full break of the lateral interactions along the whole protofilament. A drop of RQn value of native contacts for the $E(P 6)$ indicates the break of the lateral contacts along the length of 1 dimer for the P6 protofilament. Gradual increase of non-native contacts during indentation is visible for the $W(P 1)$ up to the break event. Initial increase and stabilization of the non-native contacts and then a drop to 0 is visible for W(P6). Additionally, there is a small increase in the non-native contacts just before the break of contacts on the $E(P 6)$ interface together with a small increase in the non-native contacts for $W(P 6)$. Because in this arrangement the P5/P6 interface is directly above the plate, a rapid increase in the non-native contacts for the W(P6) is not visible. N/S on both plots overlap, no change in RQn values indicate no longitudinal break. 


\begin{tabular}{ccc} 
Interface & Contacts \# & $\begin{array}{c}\text { Strength } \\
{[\mathrm{kcal} / \mathrm{mol}]}\end{array}$ \\
\hline P1/P13 & 44 & 39.60 \\
P1/P2 & 64 & 57.60 \\
P2/P3 & 75 & 67.50 \\
P3/P4 & 59 & 53.10 \\
P4/P5 & 77 & 69.30 \\
P5/P6 & 36 & 32.40 \\
P6/P7 & 70 & 63.00 \\
P7/P8 & 70 & 63.00 \\
P8/P9 & 82 & 73.80 \\
P9/P10 & 53 & 47.70 \\
P10/P11 & 56 & 50.40 \\
P11/P12 & 63 & 56.70 \\
P12/P13 & 92 & 82.80
\end{tabular}

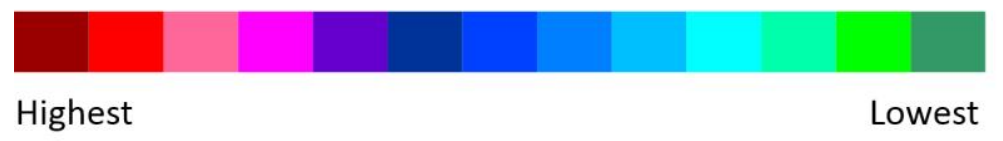

Figure S21 List of the total number of native contacts per lateral interface for each of the 12 interfaces in a 13 protofilaments MT filament (middle column), and the total strength of the interface for 1 dimer length (based on $0.90 \mathrm{kcal} / \mathrm{mol}$ per lateral contact) (left column). Color bars indicate the relative order of contacts (from the interface with the highest energy to the interface with the lowest energy). 


\section{References:}

[1] Phillips, J. C.; Hardy, D. J.; Maia, J. D. C.; Stone, J. E.; Ribeiro, J. V.; Bernardi, R. C.; Buch, R.; Fiorin, G.; Henin, J.; Jiang, W., McGreevy, R.; Melo, M. C. R.; Radak, B. K.; Skeel, R. D.; Singharoy, A.; Wang, Y.; Roux, B.; Aksimentiev, A.; Luthey-Schulten, Z.; Kale, L. V.; Schulten, K.; Chipot, C.; Tajkhorshid, E. Scalable Molecular Dynamics on CPU and GPU Architectures with NAMD, J. Chem. Phys., 2020, 153, 044130

[2] Tanner, D. E.; Chan, K.-Y.; Phillips, J. C.; Schulten, K. Parallel Generalized Born Implicit Solvent Calculations with NAMD, J. Chem. Theory. Comput., 2011, 7(11), 3635-3642

[3] Szatkowski, L.; Merz Jr, D. R.; Jiang, N.; Ejikeme, I.; Belonogov, L.; Ross, J. L.; Dima, R. I. Mechanics of the Microtubule Seam Interface Probed by Molecular Simulations and in Vitro Severing Experiments, J. Phys. Chem. B, 2019, 123, 48884900 